$1-1-2009$

\title{
Differential cross sections and spin density matrix elements for the reaction $y p \rightarrow p \omega$
}

M. Williams

Angela Biselli

Fairfield University, abiselli@fairfield.edu

CLAS Collaboration

Follow this and additional works at: https://digitalcommons.fairfield.edu/physics-facultypubs

Copyright American Physical Society Publisher final version available at http://prc.aps.org/ abstract/PRC/v80/i6/e065208

\section{Peer Reviewed}

\section{Repository Citation}

Williams, M.; Biselli, Angela; and CLAS Collaboration, "Differential cross sections and spin density matrix elements for the reaction $\mathrm{y} p \rightarrow \mathrm{p \omega}$ " (2009). Physics Faculty Publications. 46.

https://digitalcommons.fairfield.edu/physics-facultypubs/46

\section{Published Citation}

M. Williams et al. [CLAS Collaboration], "Differential cross sections and spin density matrix elements for the reaction $y p \rightarrow p \omega, "$ Physical Review C 80.6 (2009) DOI: 10.1103/PhysRevC.80.065208

This item has been accepted for inclusion in DigitalCommons@Fairfield by an authorized administrator of DigitalCommons@Fairfield. It is brought to you by DigitalCommons@Fairfield with permission from the rightsholder(s) and is protected by copyright and/or related rights. You are free to use this item in any way that is permitted by the copyright and related rights legislation that applies to your use. For other uses, you need to obtain permission from the rights-holder(s) directly, unless additional rights are indicated by a Creative Commons license in the record and/or on the work itself. For more information, please contact digitalcommons@fairfield.edu. 


\section{Differential cross sections and spin density matrix elements for the reaction $\gamma p \rightarrow p \omega$}

M. Williams, ${ }^{1, *}$ D. Applegate,,${ }^{1,}$ M. Bellis, ${ }^{1}$ C. A. Meyer, ${ }^{1}$ K. P. Adhikari,${ }^{27}$ M. Anghinolfi, ${ }^{18}$ H. Baghdasaryan,,${ }^{27,36}$ J. Ball, ${ }^{7}$ M. Battaglieri, ${ }^{18}$ I. Bedlinskiy, ${ }^{21}$ B. L. Berman, ${ }^{14}$ A. S. Biselli, ${ }^{1,11}$ C. Bookwalter, ${ }^{13}$ W. J. Briscoe, ${ }^{14}$ W. K. Brooks, ${ }^{33,35}$ V. D. Burkert, ${ }^{33}$ S. L. Careccia,${ }^{27}$ D. S. Carman, ${ }^{33}$ P. L. Cole,${ }^{16}$ P. Collins, ${ }^{3}$ V. Crede,,${ }^{13}$ A. D' Angelo,,${ }^{19,30}$ A. Daniel, ${ }^{26}$ R. De Vita, ${ }^{18}$ E. De Sanctis, ${ }^{17}$ A. Deur, ${ }^{33}$ B. Dey, ${ }^{1}$ S. Dhamija, ${ }^{12}$ R. Dickson, ${ }^{1}$ C. Djalali, ${ }^{32}$ G. E. Dodge,${ }^{27}$ D. Doughty,${ }^{8,33}$ M. Dugger, ${ }^{3}$ R. Dupre, ${ }^{2}$ A. El Alaoui, ${ }^{20, \$}$ L. Elouadrhiri, ${ }^{33}$ P. Eugenio, ${ }^{13}$ G. Fedotov,${ }^{31}$ S. Fegan, ${ }^{15}$ A. Fradi,${ }^{20}$ M. Y. Gabrielyan, ${ }^{12}$ M. Garçon, ${ }^{7}$ N. Gevorgyan, ${ }^{38}$ G. P. Gilfoyle,${ }^{29}$ K. L. Giovanetti, ${ }^{22}$ F. X. Girod,,${ }^{7,}$ W. Gohn, ${ }^{9}$ E. Golovatch, ${ }^{31}$ R. W. Gothe, ${ }^{32}$ K. A. Griffioen, ${ }^{37}$ M. Guidal, ${ }^{20}$ L. Guo, ${ }^{33, \|}$ K. Hafidi, ${ }^{2}$ H. Hakobyan,,${ }^{35,38}$ C. Hanretty, ${ }^{13}$ N. Hassall, ${ }^{15}$ K. Hicks, ${ }^{26}$ M. Holtrop, ${ }^{24}$ Y. Ilieva, ${ }^{14,32}$ D. G. Ireland, ${ }^{15}$ B. S. Ishkhanov, ${ }^{31}$ E. L. Isupov, ${ }^{31}$ S. S. Jawalkar, ${ }^{37}$ H. S. Jo, ${ }^{20}$ J. R. Johnstone, ${ }^{15}$ K. Joo, ${ }^{9}$ D. Keller ${ }^{26}$ M. Khandaker, ${ }^{25}$ P. Khetarpal,${ }^{28}$ W. Kim, ${ }^{23}$ A. Klein, ${ }^{27, \|}$ F. J. Klein, ${ }^{6}$ Z. Krahn, ${ }^{1,}$ V. Kubarovsky, ${ }^{28,33}$ S. V. Kuleshov, ${ }^{21,35}$ V. Kuznetsov, ${ }^{23}$ K. Livingston, ${ }^{15}$ H. Y. Lu, ${ }^{32}$ M. Mayer, ${ }^{27}$ J. McAndrew, ${ }^{10}$ M. E. McCracken, ${ }^{1}$ B. McKinnon, ${ }^{15}$ K. Mikhailov,${ }^{21}$ M. Mirazita, ${ }^{17}$ V. Mokeev,${ }^{31,33}$ B. Moreno,,${ }^{20}$ K. Moriya,${ }^{1}$ B. Morrison, ${ }^{3}$ H. Moutarde, ${ }^{7}$ E. Munevar, ${ }^{14}$ P. Nadel-Turonski, ${ }^{6}$ C. S. Nepali, ${ }^{27}$ S. Niccolai, ${ }^{20}$ G. Niculescu, ${ }^{22}$ I. Niculescu, ${ }^{22}$ M. R. Niroula, ${ }^{27}$ R. A. Niyazov, ${ }^{28,33}$ M. Osipenko, ${ }^{18}$ A. I. Ostrovidov, ${ }^{13}$ M. Paris,,${ }^{33, * *}$ K. Park, ${ }^{23,32,8}$ S. Park,,${ }^{13}$ E. Pasyuk, ${ }^{3}$ S. Anefalos Pereira, ${ }^{17}$ Y. Perrin ${ }^{20, t}$ S. Pisano, ${ }^{20}$ O. Pogorelko, ${ }^{21}$ S. Pozdniakov ${ }^{21}$ J. W. Price, ${ }^{4}$ S. Procureur, ${ }^{7}$ D. Protopopescu,${ }^{15}$ B. A. Raue, ${ }^{12,33}$ G. Ricco, ${ }^{18}$ M. Ripani, ${ }^{18}$ B. G. Ritchie, ${ }^{3}$ G. Rosner ${ }^{15}$ P. Rossi, ${ }^{17}$ F. Sabatié, ${ }^{7}$ M. S. Saini, ${ }^{13}$ J. Salamanca, ${ }^{16}$ C. Salgado, ${ }^{25}$ D. Schott, ${ }^{12}$ R. A. Schumacher, ${ }^{1}$ H. Seraydaryan, ${ }^{27}$ Y. G. Sharabian, ${ }^{33}$ E. S. Smith,${ }^{33}$ D. I. Sober, ${ }^{6}$ D. Sokhan,${ }^{10}$ S. S. Stepanyan, ${ }^{23}$ P. Stoler ${ }^{28}$ I. I. Strakovsky, ${ }^{14}$ S. Strauch,,${ }^{14,32}$ M. Taiuti, ${ }^{18}$ D. J. Tedeschi, ${ }^{32}$ S. Tkachenko, ${ }^{27}$ M. Ungaro, ${ }^{9,28}$ M. F. Vineyard, ${ }^{34}$ E. Voutier,,${ }^{20, \ddagger}$ D. P. Watts, ${ }^{15, \dagger}$ L. B. Weinstein,,${ }^{27}$ D. P. Weygand, ${ }^{33}$ M. H. Wood, ${ }^{5,32}$ J. Zhang, ${ }^{27}$ and B. Zhao ${ }^{9, \$ \ddagger}$

(CLAS Collaboration)

${ }^{1}$ Carnegie Mellon University, Pittsburgh, Pennsylvania 15213, USA

${ }^{2}$ Argonne National Laboratory, Argonne, Illinois 60441, USA

${ }^{3}$ Arizona State University, Tempe, Arizona 85287-1504, USA

${ }^{4}$ California State University, Dominguez. Hills, Carson, California 90747, USA

${ }^{5}$ Canisius College, Buffalo, New York 14208, USA

${ }^{6}$ Catholic University of America, Washington, DC 20064, USA

${ }^{7}$ CEA, Centre de Saclay, Irfu/Service de Physique Nucléaire, F-91191 Gif-sur-Yvette, France

${ }^{8}$ Christopher Newport University, Newport News, Virginia 23606, USA

${ }^{9}$ University of Connecticut, Storrs, Connecticut 06269, USA

${ }^{10}$ Edinburgh University, Edinburgh EH9 3JZ, United Kingdom

${ }^{11}$ Fairfield University, Fairfield Connecticut 06824, USA

${ }^{12}$ Florida International University, Miami, Florida 33199, USA

${ }^{13}$ Florida State University, Tallahassee, Florida 32306, USA

${ }^{14}$ The George Washington University, Washington, DC 20052, USA

${ }^{15}$ University of Glasgow, Glasgow G12 8QQ, United Kingdom

${ }^{16}$ Idaho State University, Pocatello, Idaho 83209, USA

${ }^{17}$ INFN, Laboratori Nazionali di Frascati, I-00044 Frascati, Italy

${ }^{18}$ INFN, Sezione di Genova, I-16146 Genova, Italy

${ }^{19}$ INFN, Sezione di Roma Tor Vergata, I-00133 Rome, Italy

${ }^{20}$ Institut de Physique Nucléaire ORSAY, Orsay, France

${ }^{21}$ Institute of Theoretical and Experimental Physics, Moscow RU-117259, Russia

${ }^{22}$ James Madison University, Harrisonburg, Virginia 22807, USA

${ }^{23}$ Kyungpook National University, Daegu 702-701, Republic of Korea

${ }^{24}$ University of New Hampshire, Durham, New Hampshire 03824-3568, USA

${ }^{25}$ Norfolk State University, Norfolk, Virginia 23504, USA

${ }^{26}$ Ohio University, Athens, Ohio 45701, USA

${ }^{27}$ Old Dominion University, Norfolk, Virginia 23529, USA

${ }^{28}$ Rensselaer Polytechnic Institute, Troy, New York 12180-3590, USA

${ }^{29}$ University of Richmond, Richmond, Virginia 23173, USA

${ }^{30}$ Universita' di Roma Tor Vergata, I-00133 Rome, Italy

${ }^{31}$ Skobeltsyn Nuclear Physics Institute, Moscow RU-119899, Russia

${ }^{32}$ University of South Carolina, Columbia, South Carolina 29208, USA

${ }^{33}$ Thomas Jefferson National Accelerator Facility, Newport News, Virginia 23606, USA

${ }^{34}$ Union College, Schenectady, New York 12308, USA

${ }^{35}$ Universidad Técnica Federico Santa María, Casilla 110-V Valparaíso, Chile

${ }^{36}$ University of Virginia, Charlottesville, Virginia 22901, USA 


\author{
${ }^{37}$ College of William and Mary, Williamsburg, Virginia 23187-8795, USA \\ ${ }^{38}$ Yerevan Physics Institute, 375036 Yerevan, Armenia \\ (Received 23 August 2009; published 30 December 2009)
}

\begin{abstract}
High-statistics differential cross sections and spin-density matrix elements for the reaction $\gamma p \rightarrow p \omega$ have been measured using the CEBAF large acceptance spectrometer (CLAS) at Jefferson Lab for center-of-mass (c.m.) energies from threshold up to $2.84 \mathrm{GeV}$. Results are reported in $11210-\mathrm{MeV}$ wide c.m. energy bins, each subdivided into $\cos \theta_{\mathrm{c} . \mathrm{m} \text {. }}^{\omega}$ bins of width 0.1 . These are the most precise and extensive $\omega$ photoproduction measurements to date. A number of prominent structures are clearly present in the data. Many of these have not previously been observed due to limited statistics in earlier measurements.
\end{abstract}

DOI: 10.1103/PhysRevC.80.065208

\section{INTRODUCTION}

Studying low-energy $\omega$ photoproduction presents an interesting opportunity to search for new baryon resonances. Previous experiments have produced cross-section measurements with relatively high precision at most production angles; however, precise spin-density matrix elements have only been measured at very forward angles [1-5]. Theoretical interpretation of these data indicate strong $t$-channel contributions from both $\pi^{0}$ and Pomeron exchange, while the backward peak in the cross section has been interpreted as evidence of nucleon $u$-channel contributions [6-9]. Several attempts have been made to extract resonant contributions that have obtained conflicting results [8,10-14]. Precise polarization information is needed in order to place stringent constraints on the physics interpretation of $\omega$ photoproduction data.

The impact of polarization information can be seen by comparing the partial wave analysis results obtained using only cross-section data [13] to those that also included the low-precision polarization results from SAPHIR [14]. The former found that at threshold the dominant $s$-channel contributions are from the $P_{13}(1720)$ and $F_{15}(1680)$, while the latter found that the $D_{15}(1675)$ and $F_{15}(1680)$ are dominant in this region. Including polarization information, even with very limited precision, provided strong additional constraints on the interpretation of the data. Thus, obtaining high-precision polarization results is a vital step toward understanding baryon resonance contributions to $\omega$ photoproduction.

\footnotetext{
*Present address: Imperial College London, London SW7 2AZ, United Kingdom.

†Present address: Stanford University, Stanford, CA 94305, USA.

${ }^{\ddagger}$ Present address: LPSC-Grenoble, France.

§Present address: Thomas Jefferson National Accelerator Facility,

Newport News, Virginia 23606, USA.

"Present address: Los Alamos National Laborotory, New Mexico, NM, USA.

IPresent address: University of Minnesota, Minneapolis, MN 55455, USA.

*** Present address: The George Washington University, Washington, DC 20052, USA.

${ }^{\dagger}$ Present address: Edinburgh University, Edinburgh EH9 3JZ, United Kingdom.

${ }^{\ddagger}$ Present address: College of William and Mary, Williamsburg, Virginia 23187, USA.
}

PACS number(s): 11.80.Cr, 11.80.Et, 13.30.Eg, 14.20.Gk

Beyond this, quark model calculations of baryon decays [15] predict that a number of the so-called missing baryons should couple to $\omega N$ final states. In particular, in the above model, nearly all of the missing positive parity $N^{*}$ states are expected to have non-negligible couplings to $\omega N$. Thus, good data on $\omega$ photoproduction coupled with a partial wave analysis (PWA) could provide important new information on lightquark baryons.

The data presented here are part of a larger program to simultaneously measure photoproduction of mesons and then carry out partial wave analyses on the resulting data. This article presents differential cross section and spin density matrix element measurements for $\omega$ photoproduction. In a companion article published concurrent to this one [16], we present a detailed partial wave analysis of these data where clear $s$-channel resonance contributions are identified. A forthcoming article will discuss the impact on current theoretical models and coupled-channel analyses of these new precise measurements [17].

\section{EXPERIMENTAL SETUP}

The data were obtained using the CEBAF large acceptance spectrometer (CLAS) housed in Hall B at the Thomas Jefferson National Accelerator Facility. Real photons were produced via bremsstrahlung from a $4 \mathrm{GeV}$ electron beam hitting a $10^{-4}$ radiation length gold foil. The recoiling electrons were then analyzed using a dipole magnet and scintillator hodoscopes in order to obtain, or "tag," the energy of the photons [18] (the so-called photon tagger). The tagging range and energy resolution were $20 \%-95 \%$ and $0.1 \%$ of the electron beam energy, respectively. The useful center-of-mass (c.m.) energy $(W)$ range for this analysis was from $\omega$-photoproduction threshold at $W=1.72 \mathrm{GeV}$ up to $2.84 \mathrm{GeV}$. In this range, the data were analyzed in $10-\mathrm{MeV}$ wide $W$ bins.

The physics target, which was filled with liquid hydrogen, was a $40-\mathrm{cm}$ long cylinder with a radius of $2 \mathrm{~cm}$. Continuous monitoring of the temperature and pressure permitted determination of the density with uncertainty of $0.2 \%$. The target cell was surrounded by 24 "start counter" scintillators that were used in the event trigger.

The CLAS detector utilized a nonuniform toroidal magnetic field of peak strength near $1.8 \mathrm{~T}$ in conjunction with drift chamber tracking to determine particle momenta. The detector was divided into six sectors, such that when viewed along the beam line it was sixfold symmetric. Charged particles with 
laboratory polar angles in the range $8^{\circ}-140^{\circ}$ could be tracked over approximately $83 \%$ of the azimuthal angle. A set of 288 scintillators placed outside of the magnetic field region were used in the event trigger and during offline analysis in order to determine time of flight (TOF). The momentum resolution of the detector was, on average, about $0.5 \%$. Other components of the CLAS, such as the Cerenkov counters and the electromagnetic calorimeters, were not used in this analysis. A more detailed description of the CLAS can be found in Ref. [19].

The event trigger required a coincidence between signals from the photon tagger and the CLAS. The signal from the tagger consisted of an OR of the first 40 of the 61 total timing scintillators, corresponding to photon energies above $1.5 \mathrm{GeV}$. Recording of events associated with photons hitting counters 41-61 required a random tagger hit in counters 1-40 during the trigger timing window. This allowed for the acquisition of greater statistics at the higher photon-energy range of this experiment. The signal from the CLAS required at least two sector-based signals. These signals consisted of an OR of any of the four start counter scintillators in coincidence with an OR of any of the 48 time-of-flight scintillators in the sector. The rate at which hadronic events were accumulated was about $5 \mathrm{kHz}$; however, only a small fraction of these events contained the reaction of interest to the analysis presented here.

\section{DATA AND EVENT SELECTION}

The data used were obtained in the summer of 2004 during the CLAS "g11a" data taking period, in which approximately 20 billion triggers were recorded. The relatively loose electronic trigger led to accumulation of data for a number of photoproduction reactions. The relative timing of the photon tagger, the start counter and the time-of-flight elements were aligned during offline calibration. Calibrations were also made for the drift times of each of the drift chamber packages and the pulse heights of each of the time-of-flight counters. Finally, processing of the raw data was performed in order to reconstruct tracks in the drift chambers and match them with time-of-flight counter hits.

The reconstructed tracks were corrected for small imperfections in the magnetic field map and drift chamber alignment, along with their mean energy losses as they passed through the target, the beam pipe, the start counter and air. In addition, small corrections were made to the incident photon energies to account for mechanical sag in the tagger hodoscope.

The CLAS was optimized for detection of charged particles; thus, the $\pi^{+} \pi^{-} \pi^{0}$ decay of the $\omega$ was used to select the reaction of interest in this analysis. Detection of two positively charged particles and one negatively charged particle was required. A cut was placed on the confidence levels obtained from one-constraint kinematic fits performed to the hypothesis $\gamma p \rightarrow p \pi^{+} \pi^{-}\left(\pi^{0}\right)$ in order to select events consistent with a missing $\pi^{0}$. All negatively charged tracks were assigned a $\pi^{-}$identification. Kinematic fits were run for each of the possible $p, \pi^{+}$particle assignments for the positively charged tracks using each of the recorded photons in the event. The combinations of photons and charged particles with confidence levels greater than $10 \%$ were retained for further analysis.

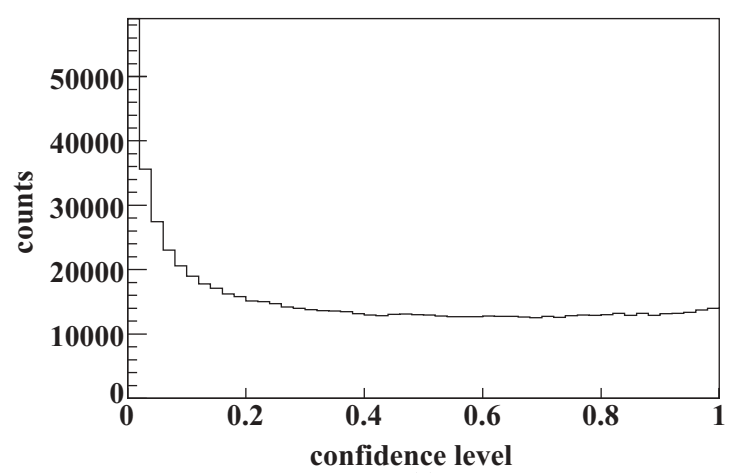

(a)

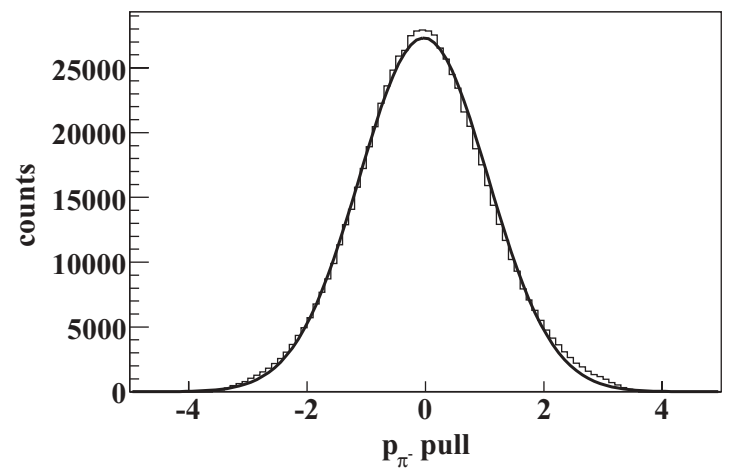

(b)

FIG. 1. (a) The confidence levels resulting from four-constraint kinematic fits performed on a sample of events to the hypothesis $\gamma p \rightarrow p \pi^{+} \pi^{-}$integrated over all kinematics. The "peak" near zero consists of events that do not match the hypothesis, along with poorly measured (due to multiple scattering, etc.) signal events. Agreement with the ideal (flat) distribution for signal events is very good. (b) Example pull-distribution for the momentum of the $\pi^{-}$from the same kinematic fits as in (a). Only events with a confidence level larger than $1 \%$ are shown. The line represents a Gaussian fit to this distribution. For this event sample, the parameters obtained are $\mu=-0.029 \pm 0.001, \sigma=1.086 \pm 0.001$ (the uncertainties are purely statistical), which are in very good agreement with the ideal values $\mu=0, \sigma=1$. Both (a) and (b) are good indicators that the CLAS error matrix is well understood.

The covariance matrix was studied using four-constraint kinematic fits (energy and momentum conservation imposed) performed on the exclusive reaction $\gamma p \rightarrow p \pi^{+} \pi^{-}$in both real and Monte Carlo data samples. The confidence levels in all kinematic regions were found to be sufficiently flat and the pull-distributions (stretch functions) were Gaussians centered at zero with $\sigma=1$ (see Fig. 1). The uncertainty in the extracted yields due to differences in signal lost because of this confidence-level cut in real as compared to Monte Carlo data is estimated to be $3 \%-4 \%$.

The tagger signal time, which was synchronized with the accelerator radio-frequency (RF) timing, was propagated to the reaction vertex in order to obtain the start time for the event. The stop time for each track was obtained from the TOF scintillator element hit by the track. The difference between these two times was the measured time of flight, $t_{\text {meas }}$. Track reconstruction through the CLAS magnetic field yielded both the momentum, $\vec{p}$, of each track, along with the path length, $L$, from the reaction vertex to the time-of-flight counter hit by 


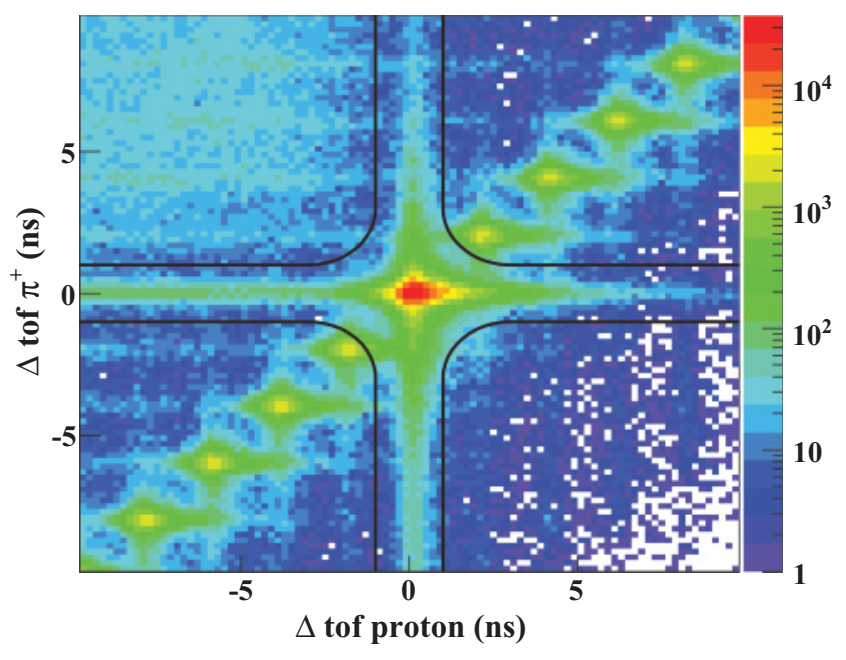

FIG. 2. (Color online) $\Delta$ tof $_{\pi^{+}}(\mathrm{ns})$ versus $\Delta \operatorname{tof}_{p}(\mathrm{~ns})$ : Particle identification cut for a sample of events that pass a $10 \%$ confidence level cut when kinematically fit to the hypothesis $\gamma p \rightarrow p \pi^{+} \pi^{-}\left(\pi^{0}\right)$. The black lines indicate the timing cuts. Note the logarithmic scale on the intensity axis.

the track. The expected time of flight for a mass hypothesis, $m$, is then given by

$$
t_{\exp }=\frac{L}{c} \sqrt{1+\left(\frac{m}{p}\right)^{2}} \text {. }
$$

The difference in these two time-of-flight calculations, $\Delta$ tof $=$ $t_{\text {meas }}-t_{\text {exp }}$, was used in order to separate protons from pions and to remove events associated with out-of-time photons.

Figure 2 shows $\Delta$ tof for the track passing the kinematic fit under the $\pi^{+}$hypothesis versus $\Delta$ tof for the track passing the fit under the proton hypothesis. The region near $(0,0)$ contains events where both tracks are good matches to their respective particle identification hypotheses. The 2-ns radio-frequency time structure of the accelerator is evident in the out-of-time event clusters. Events outside of the black lines, where neither hypothesis was met, were cut from our analysis. This cut was designed to remove a minimal amount of good events. The Feldman-Cousins method [20] was used to place an upper limit on the signal lost at $1.3 \%$. Any remaining accidental events fell into the broad background under the $\omega$, and were rejected during the signal-background separation stage of the analysis discussed in Sec. IV.

Fiducial cuts were applied on the momenta and angles of the tracks in order to select events from the well-understood regions of the detector. Included in these cuts was the removal of 13 of the 288 time-of-flight elements due to poor performance. In addition, events where the missing $\pi^{0}$ was moving along the beam line, $\cos \theta_{\mathrm{c} . \mathrm{m} .}^{\pi^{0}}>0.99$, were cut in order to remove leakage from the $\gamma p \rightarrow p \pi^{+} \pi^{-}$reaction. A more detailed description of the analysis procedures presented in this article can be found in Ref. [21].

\section{SIGNAL-BACKGROUND SEPARATION}

In addition to $\omega$ events, the resulting sample consisted of events from the reaction $\gamma p \rightarrow p \pi^{+} \pi^{-} \pi^{0}$ where the three-pion invariant mass was consistent with that of the $\omega$. These background events could arise from a variety of physics reactions, but they all share the characteristic that they cannot reproduce the narrow mass structure associated with the $\omega$. Typical background levels were $5 \%$ to $10 \%$ of the $\omega$ peak height, while in a small number of bins near threshold and where the cross section is very low (e.g., near $\cos \theta_{\text {c.m. }}^{\omega}$ of -0.2 at the highest photon energies), the background was as large as $25 \%$ of the $\omega$ peak. Thus, the three-pion invariant mass could be used as a tool to separate the signal from the background.

In order to accurately extract physical observables for $\omega$ photoproduction, background events (all non- $\omega$ events) must be separated from the signal in a way that preserves all kinematic correlations. The method we have developed, described in detail elsewhere [21,22], was used to assign each event a signal weight factor, $Q$, or equivalently, a background weight factor, $1-Q$. These $Q$ factors were then used to weight each event's contribution to the "log likelihood" during the event-based fits discussed in Sec. V. These fits were used to obtain the detector acceptance and to extract the spin density matrix elements. The $Q$ factors were also used to weight each event's contribution to the differential cross section.

The key feature of our procedure involves selecting each event's $N_{c}$ "nearest neighbor" events (we chose $N_{c}=500$ ). This is done by defining a metric in the space of all relevant kinematic variables, excluding the $3 \pi$ invariant mass, $M_{3 \pi}$. Each subset of events occupies a very small region of phase space; thus, the $M_{3 \pi}$ distribution can safely be used to determine each event's $Q$ factor, while preserving the correlations present in the remaining kinematic variables. This method facilitates separation of the signal and background without having to resort to dividing the data up into bins. Binning the data is undesirable due to the high dimensionality of the reaction being studied in this analysis.

To this end, unbinned maximum likelihood fits were carried out for each event, using its nearest neighbors, to determine the parameters $\vec{\alpha}=\left(b_{0}, b_{1}, b_{2}, b_{3}, b_{4}, s, \sigma\right)$ in the probability density function

$$
F\left(M_{3 \pi}, \vec{\alpha}\right)=\frac{B\left(M_{3 \pi}, \vec{\alpha}\right)+S\left(M_{3 \pi}, \vec{\alpha}\right)}{\int\left[B\left(M_{3 \pi}, \vec{\alpha}\right)+S\left(M_{3 \pi}, \vec{\alpha}\right)\right] d M_{3 \pi}},
$$

where

$$
S\left(M_{3 \pi}, \vec{\alpha}\right)=s \cdot V\left(M_{3 \pi}, M_{\omega}, \Gamma_{\omega}, \sigma\right)
$$

parametrizes the signal as a Voigtian (convolution of a BreitWigner and a Gaussian) with mass $M_{\omega}=0.78256 \mathrm{GeV} / c^{2}$, natural width $\Gamma_{\omega}=0.00844 \mathrm{GeV} / c^{2}$ and resolution $\sigma$. The parameter $s$ sets the overall strength of the signal. The background in each small phase space region was parametrized as a fourth order polynomial,

$$
B\left(M_{3 \pi}, \vec{\alpha}\right)=b_{4} M_{3 \pi}^{4}+b_{3} M_{3 \pi}^{3}+b_{2} M_{3 \pi}^{2}+b_{1} M_{3 \pi}+b_{0}
$$


The $Q$ factor for the event was then calculated as

$$
Q_{i}=\frac{S\left(M_{3 \pi}^{i}, \hat{\alpha}_{i}\right)}{S\left(M_{3 \pi}^{i}, \hat{\alpha}_{i}\right)+B\left(M_{3 \pi}^{i}, \hat{\alpha}_{i}\right)},
$$

where $M_{3 \pi}^{i}$ is the event's $3 \pi$ invariant mass and $\hat{\alpha}_{i}$ are the estimators for the parameters obtained from the $i$ th event's fit. The signal yield could then be obtained in any kinematic bin as

$$
\mathcal{Y}_{\omega}=\sum_{i}^{N} Q_{i},
$$

where $N$ is the number of events in the bin.

The full covariance matrix obtained from each fit was used to obtain the uncertainty in $Q, \sigma_{Q}$. This varied depending on kinematics; however, it was typically about 3\%. The uncertainty of the extracted yield, in any kinematic bin, was obtained by adding the $Q$-factor uncertainties (assuming $100 \%$ correlation) to the statistical uncertainty of the yield:

$$
\sigma_{\mathcal{Y}_{\omega}}^{2}=\sum_{i}^{N} Q_{i}^{2}+\left(\sum_{i}^{N} \sigma_{Q i}\right)^{2} \text {. }
$$

Studies were performed using various background parametrizations, including polynomials of different orders, all of which yielded results within the values obtained for $\sigma_{Q}$. Therefore, we conclude that no additional systematic uncertainty is required.

Figure 3 demonstrates the effectiveness of applying this procedure in a single center-of-mass energy bin. Figure 3(a) shows the $M_{3 \pi}$ distribution, integrated over all kinematics, and the estimated background using the procedure described above. The results are quite plausible; however, $\omega$ photoproduction provides us with a more stringent test of this procedure.

The distribution of the decay quantity $\lambda$, which can be written in terms of the pion momenta in the $\omega$ rest frame as

$$
\lambda=\frac{\left|\vec{p}_{\pi^{+}} \times \vec{p}_{\pi^{-}}\right|^{2}}{\operatorname{MAX}\left(\left|\vec{p}_{\pi^{+}} \times \vec{p}_{\pi^{-}}\right|^{2}\right)},
$$

must be linear in shape and intersect 0 at $\lambda=0$ for $\omega$ events-this follows directly from the $\omega \rightarrow \pi^{+} \pi^{-} \pi^{0}$ amplitude defined in Eq. (10). Figure 3(b) shows the $\lambda$ distribution, integrated over all kinematics, for events in the same bin shown in Fig. 3(a) in the region $\pm 25 \mathrm{MeV} / c^{2}$ around the $\omega$ peak, along with the extracted signal and background distributions. The signal distribution is well described by the function $a \lambda$. The small discrepancy near $\lambda=0$ is the result of detector resolution.

The method we have employed has cleanly separated signal from background in the quantity $\lambda$, even though the known linear behavior of the signal was not enforced in the fits. In fact, this method has effectively separated signal from background in all distributions, successfully preserving all kinematic correlations.

A detailed study of the systematic biases of the background subtraction technique was carried out as part of this analysis. Not only was the function that was used to parametrize the background varied, but the number of nearest neighbor events was varied over a wide range and several different metrics

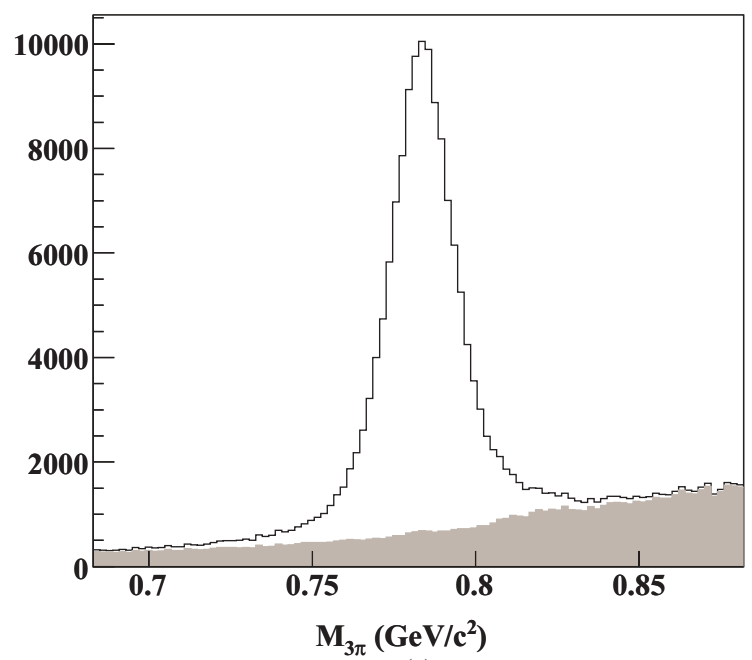

(a)

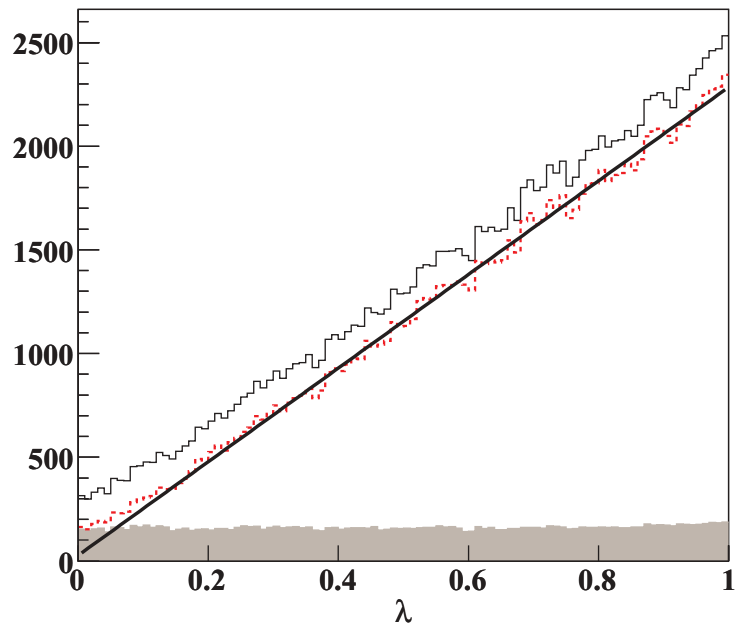

(b)

FIG. 3. (Color online) (a) The $3 \pi$ invariant mass distribution in the $W=2.205 \mathrm{GeV}$ bin, integrated over all kinematics, for all events (unshaded) and for events weighted by the background factors, $1-Q$ (shaded). (b) The $\lambda$ distribution of events in the same $W$ bin that satisfy $\left|M_{3 \pi}-M_{\omega}\right|<25 \mathrm{MeV} / c^{2}$ (unshaded), the same events weighted by signal factors $Q$ (red, dashed), and by background factors $1-Q$ (shaded). The line represents a fit of the signal to the function $a \lambda$.

were used to determine the nearest neighbor events. The observed physical measurements were found to be completely insensitive to changes in these parameters over any reasonable set of values. Because of this, we associate no additional systematic error with these choices. A detailed description of this study is contained in Ref. [22].

For energy bins near threshold and for "edge" regions (i.e., forward- and backward-most angles) in some energy bins, the lack of events on both sides of the peak leaves the fits unconstrained. In these regions, the energy dependence of the $Q$ factors obtained in the closest energy bins for which fitting could be used were projected down to the regions in question in order to obtain the $Q$ factors. Figure 4 shows the results of this procedure in the $W=1.735 \mathrm{GeV}$ bin. By studying the $\lambda$ distributions in these bins, the systematic uncertainty associated with the projected $Q$ factors in the edge regions is 


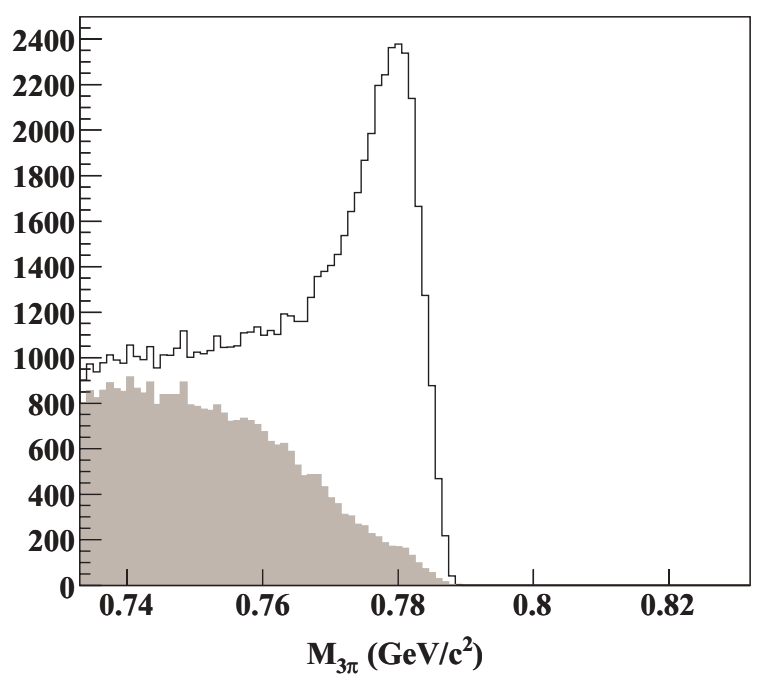

FIG. 4. The $3 \pi$ invariant mass distribution in the $W=1.735 \mathrm{GeV}$ bin, integrated over all kinematics, for all events (unshaded) and for events weighted by the background factors, $1-Q$ (shaded).

estimated to be $5 \%$. In the first two energy bins above threshold, the uncertainties are estimated to be $15 \%$ and $10 \%$ for the $W=1.725 \mathrm{GeV}$ and $1.735 \mathrm{GeV}$ bins, respectively.

\section{ACCEPTANCE}

The efficiency of the detector was modeled using the standard CLAS GEANT-based simulation package and the Monte Carlo technique. A total of 200 million events were generated pseudorandomly, sampled from a phase space distribution. Each particle was propagated from the event vertex through the CLAS resulting in a simulated set of detector signals for each track. The simulated events were then processed using the same reconstruction software as the data. In order to account for the event trigger used in this experiment (see Sec. II), a study was performed to obtain the probability of a track satisfying the sector-based coincidences required by the trigger as a function of kinematics and struck detector elements. The average effect of this correction in our analysis, which requires three detected particles, is about 5\%-6\%.

An additional momentum smearing algorithm was applied in order to better match the resolution of the Monte Carlo to that of the data. Its effects were studied using four-constraint kinematic fits performed on simulated $\gamma p \rightarrow p \pi^{+} \pi^{-}$events. After applying the momentum smearing algorithm, the same covariance matrix used for the data also produced flat confidence level distributions in all kinematic regions for the Monte Carlo data as well. The simulated $\omega$ events were then processed with the same analysis software as the data, including the one-constraint kinematic fits. At this stage, all detector and software efficiencies were accounted for.

In order to evaluate the CLAS acceptance for the $\gamma p \rightarrow p \omega$ reaction, all kinematic correlations between the final state particles must be accurately reproduced by the simulated data. Typically, this is done by using a physics model when generating Monte Carlo events. Due to the lack of any pre- existing precise polarization measurements in the kinematic regions that contain most of our data, this was not an option. Instead, we chose to expand the scattering amplitude, $\mathcal{M}$, in a very large basis of $s$-channel waves as follows:

$$
\mathcal{M}_{m_{i}, m_{\gamma}, m_{f}, m_{\omega}}(\vec{x}, \vec{\alpha}) \approx \sum_{J=\frac{1}{2}}^{\frac{21}{2}} \sum_{P= \pm} \mathcal{A}_{m_{i}, m_{\gamma}, m_{f}, m_{\omega}}^{J^{P}}(\vec{x}, \vec{\alpha}),
$$

where $\vec{\alpha}$ denotes a vector of 108 fit parameters, $\vec{x}$ denotes the complete set of kinematic variables describing the reaction, $m_{i}, m_{\gamma}, m_{f}, m_{\omega}$ are the spin projections on the incident photon direction in the center-of-mass frame, and $\mathcal{A}$ are the $s$-channel partial wave amplitudes.

The $\omega \rightarrow \pi^{+} \pi^{-} \pi^{0}$ amplitude, which is included in the $\mathcal{A}$ 's above, can be written in terms of the isovectors and the four-momenta of the pions, $\vec{I}_{\pi}$ and $p_{\pi}$, respectively, as well as the $\omega$ four-momentum $(q)$ and polarization $(\epsilon)$ as

$$
A_{\omega \rightarrow \pi^{+} \pi^{-} \pi^{0}} \propto\left[\left(\vec{I}_{\pi^{+}} \times \vec{I}_{\pi^{0}}\right) \cdot \vec{I}_{\pi^{-}}\right] \epsilon_{\mu \nu \alpha \beta} p_{\pi^{+}}^{v} p_{\pi^{-}}^{\alpha} p_{\pi^{0}}^{\beta} \epsilon^{\mu}\left(q, m_{\omega}\right),
$$

which is fully symmetric under interchange of the three pions. For this reaction, where all final states contain $\omega \rightarrow \pi^{+} \pi^{-} \pi^{0}$, the isovector triple product simply contributes a factor to the global phase of all amplitudes. In the $\omega$ rest frame, Eq. (10) simplifies to

$$
A_{\omega \rightarrow \pi^{+} \pi^{-} \pi^{0}} \propto\left(\vec{p}_{\pi^{+}} \times \vec{p}_{\pi^{-}}\right) \cdot \vec{\epsilon}\left(m_{\omega}\right) .
$$

The remaining $s$-channel structure of the amplitudes $\mathcal{A}$, as well as the details concerning the fit parameters, is described in [21].

Unbinned maximum likelihood fits were performed in each $W$ bin in order to obtain the estimators $\hat{\alpha}$ for the parameters $\vec{\alpha}$ in Eq. (9). The results of these fits were used to obtain a weight, $I_{i}$, for each Monte Carlo event according to

$$
I_{i}=\sum_{m_{i}, m_{\gamma}, m_{f}}\left|\sum_{m_{\omega}} \mathcal{M}_{m_{i}, m_{\gamma}, m_{f}, m_{\omega}}\left(\vec{x}_{i}, \hat{\alpha}_{i}\right)\right|^{2},
$$

where $\vec{x}_{i}$ is the complete set of kinematic variables of the $i^{t h}$ event. The weighted accepted Monte Carlo events fully reproduce the real data in all distributions, including all correlations (see Figs. 5 and 6). We note here that the results of these fits are not interpreted as physics, i.e., they are not considered evidence of resonance contributions to the $\omega$ photoproduction reaction. They are simply used in order to provide a complete description of the data.

The acceptance in any kinematic bin is then obtained as

$$
\operatorname{acc}(\vec{x})=\frac{\sum_{i}^{N_{\mathrm{acc}}} I_{i}}{\sum_{j}^{N_{\mathrm{gen}}} I_{j}},
$$

where $N_{\text {acc }}\left(N_{\text {gen }}\right)$ is the number of accepted (generated) Monte Carlo events in the bin and the I's are the event weights discussed above. An accurate physics generator would use the factors of $I$ during the event generation stage, rather than weighting the accepted events. The resulting acceptance calculation would be the same, modulo statistical fluctuations. 


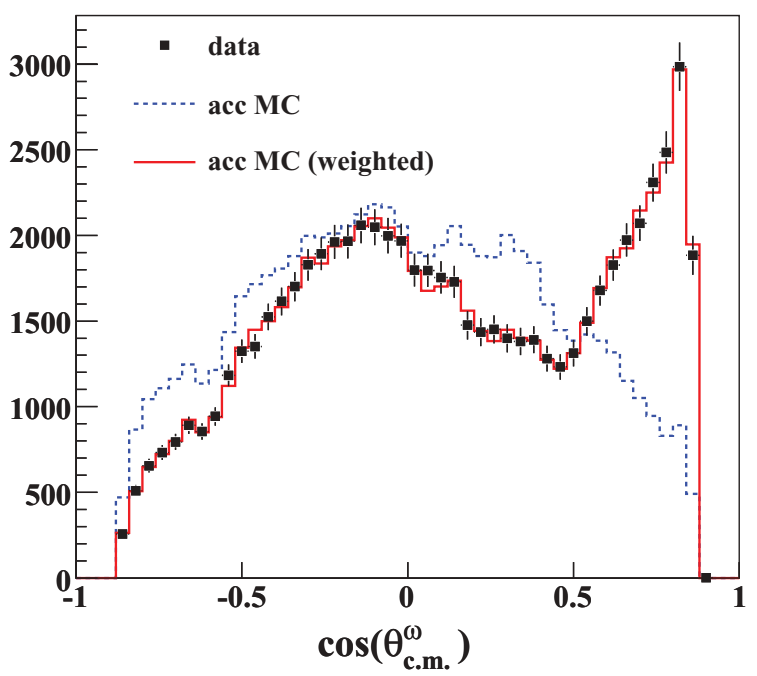

FIG. 5. (Color online) Example fit result in the $W=2.005 \mathrm{GeV}$ bin for data (black squares), phase space accepted Monte Carlo events (blue, dashed line) and phase space accepted Monte Carlo events weighted by the fits discussed in Sec. V (red, solid line). The weighted Monte Carlo provides an excellent description of the data.

The statistical uncertainties in the acceptance calculations due to the finite number of Monte Carlo events generated in each kinematic bin were obtained from the standard binomial distribution. The systematic uncertainty in the acceptance calculation is discussed in Sec. VII.

\section{NORMALIZATION}

The measured rate of electrons detected by the tagger was used to compute the number of photons incident on the target by sampling tagger hits not in coincidence with the CLAS. These rates were integrated over the live-time of the experiment in order to obtain the total photon flux associated with each tagger element. Losses of photons in the beam line due to effects such as beam collimation were determined during dedicated runs using a total-absorption counter placed downstream of the CLAS [23].

The standard electronics hardware that has traditionally been used to determine the experimental live-time malfunctioned during the g11a data taking period. A downstream device used to measure the beam current during electron runs [19] was used instead. The relatively low count rate in this device during photon running led to increased uncertainty in the live-time measurement. The stability of normalized $\omega$ yields for runs with different beam currents was used to estimate this uncertainty to be about $3 \%$.

As was stated in Sec. II, only 40 of the 61 timing elements of the photon tagger were included in the event trigger. Events associated with hits in the "untriggered" counters, 41-61, were only recorded if a random hit in counters $1-40$ occurred during the trigger time window. The electron rates used to measure the photon flux, discussed above, were used to calculate the probability of such an occurrence, $P_{\text {trig }}=0.467$. The measured flux for tagger counters $41-61$ was scaled down by $P_{\text {trig }}$ to account for the event trigger.

Defective electronics in one of the tagger channels led to inaccurate flux measurements in the energy bins at $W=2.735 \mathrm{GeV}$ and $2.745 \mathrm{GeV}$. The flux in the energy bin at $W=1.955 \mathrm{GeV}$ was also deemed unreliable due to its inclusion of events associated with both triggered and untriggered tagger counters. Differential cross sections are not reported in these three energy bins; however, spin density matrix elements, which do not require normalization information, are reported.

\section{SYSTEMATIC UNCERTAINTIES}

The $\omega$ photoproduction cross section, for the case with an unpolarized beam and an unpolarized target, must be isotropic in the azimuthal angle. Thus, the acceptance-corrected $\omega$ yields, each obtained in an individual CLAS sector, must be consistent with each other. By examining the consistency of these yields, we estimated the relative uncertainty in the acceptance correction to be between $4 \%-6 \%$, depending on center-of-mass energy. This is added in quadrature with uncertainties due to particle identification $(1.3 \%)$ and confidence level $(3 \%)$ cuts to obtain an overall estimated acceptance uncertainty of 5\%-7\%.

It is common practice in photoproduction experiments to check the quality of the normalization calculation by computing the single pion cross section and comparing it to the world's data; however, the two-track trigger used in this experiment does not permit such a calculation. In order to check our normalization, cross sections were also computed for several other reactions from the g11a data set and compared to previously published CLAS data. The run-to-run consistency of the normalized $\omega$ yield was also examined. Based on these studies, we estimate the normalization uncertainty to be $7.3 \%$. When combined with contributions from photon transmission efficiency $(0.5 \%)$ and live-time (3\%), the total estimated normalization uncertainty is $7.9 \%$.

The acceptance and normalization uncertainties discussed above were then combined with contributions from target density and length $(0.2 \%)$, along with branching fraction $(0.7 \%)$ to obtain a total uncertainty, excluding contributions from signal-background separation that are calculated "pointto-point," of about 9\%-11\%. In the first two energy bins above threshold, the additional uncertainties in the signalbackground separation method (see Sec. IV) increase this number to $13 \%-17 \%$.

\section{RESULTS}

\section{A. Differential cross sections}

Differential cross sections, $d \sigma / d \cos \theta_{\mathrm{c} . \mathrm{m} \text {. }}^{\omega}$, were computed in $10910-\mathrm{MeV}$ wide bins in $W$. Each energy bin was divided into 20 bins in $\cos \theta_{\text {c.m. }}^{\omega}$ of width 0.1 , although results could not be extracted in every bin due to limitations in the detector acceptance. In total, 1960 cross-section points are reported here. The centroid of each bin is reported as the mean of 

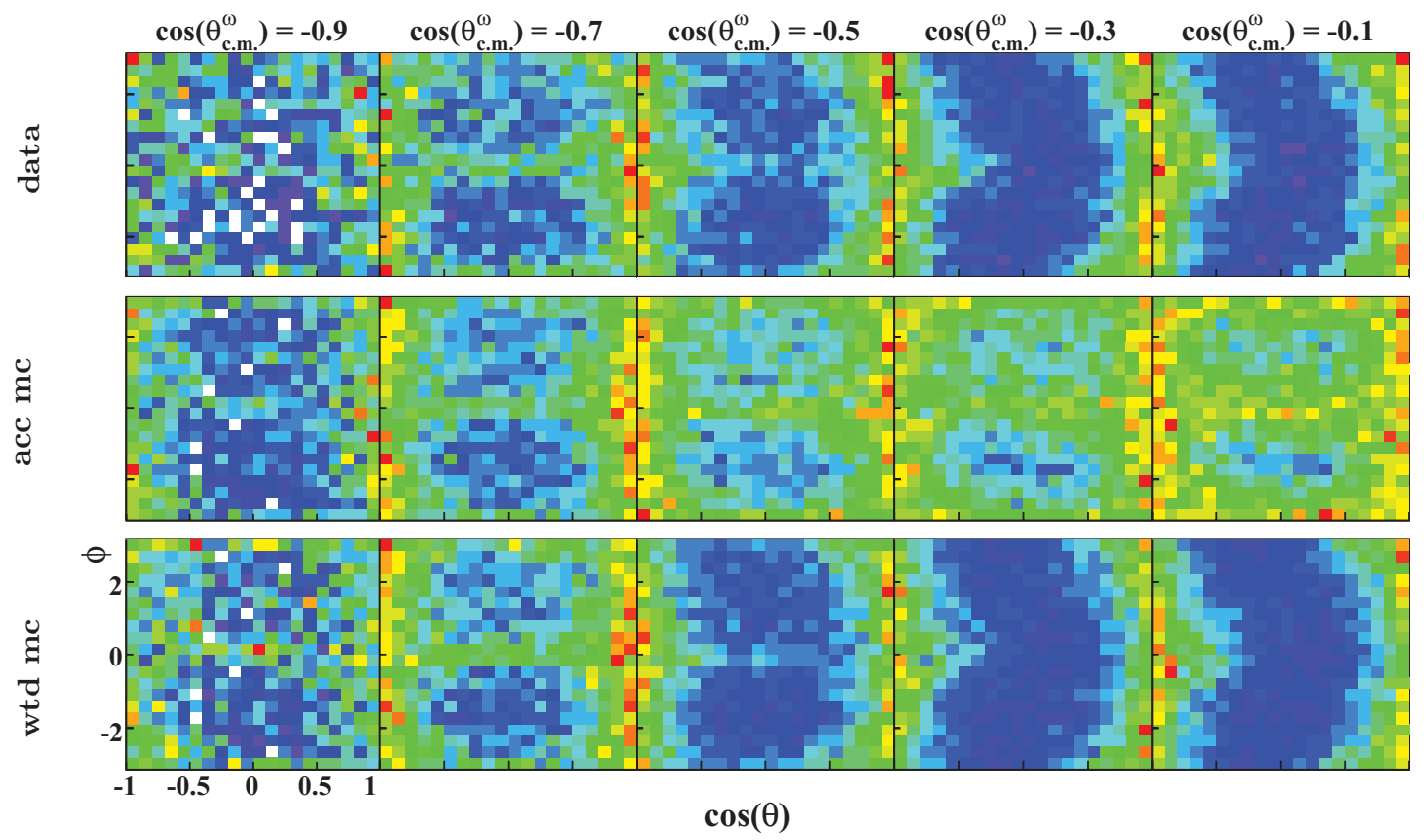

(a)
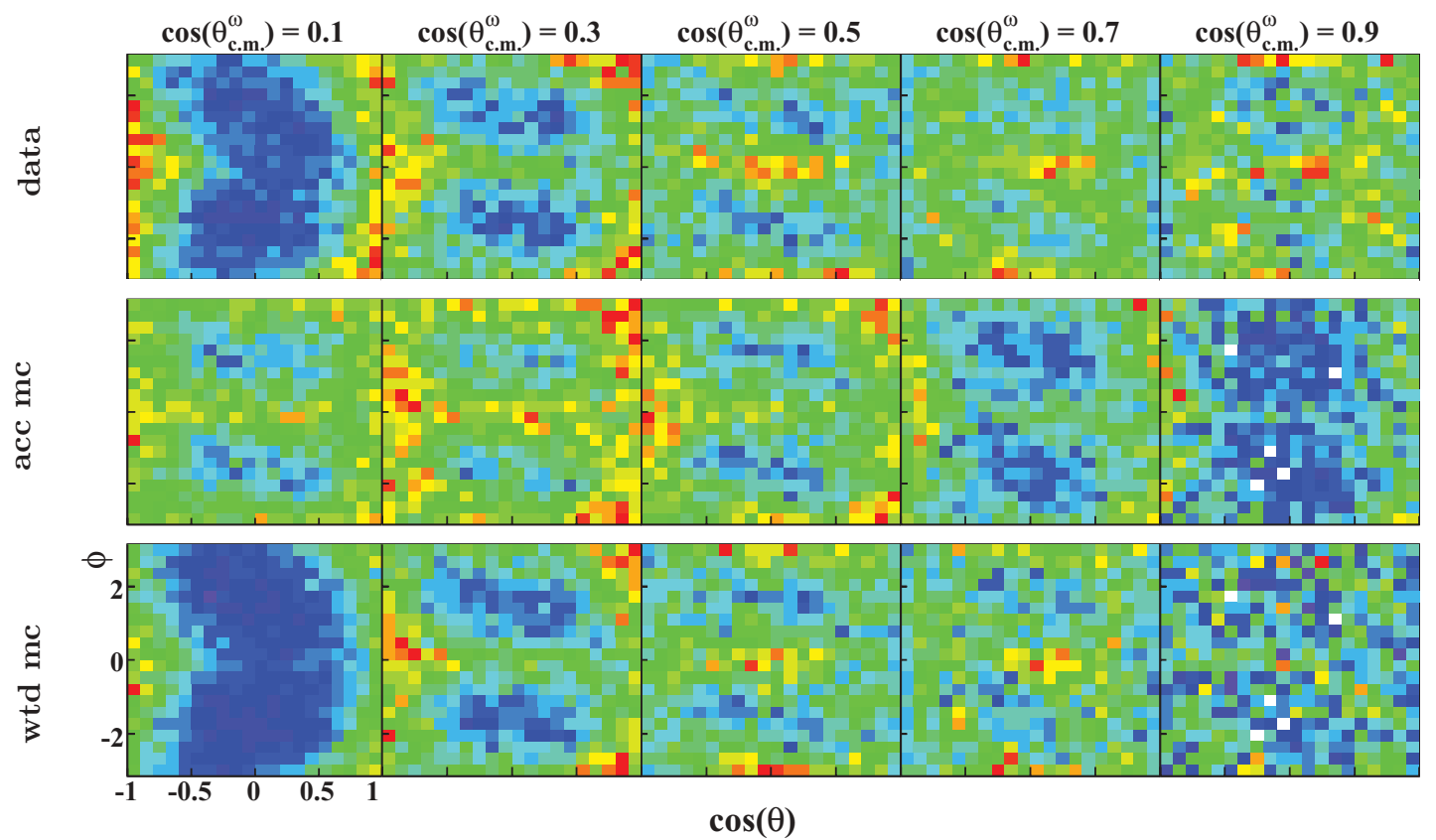

(b)

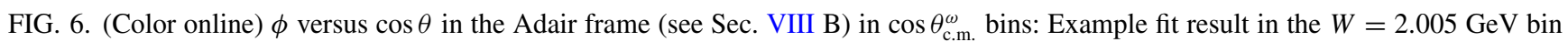
for data (top row), phase space accepted Monte Carlo events (middle row), and phase space accepted Monte Carlo events weighted by the fits discussed in Sec. V (bottom row). (a) shows backward angles, while (b) shows forward angles. Note that the weighted Monte Carlo provides an accurate description of the data $\left(\chi^{2} /\right.$ degrees of freedom $\left.\sim 0.6\right)$.

the range of the bin with nonzero acceptance. The results are shown in Figs. 7-10. The error bars contain the uncertainties of the yield extraction, discussed in Sec. IV, along with statistical uncertainties from the Monte Carlo acceptance calculations. The overall systematic uncertainty, discussed in Sec. VII, is estimated to be between $9 \%-11 \%$, depending on center-ofmass energy.
In the "transverse direction," which we can loosely define as $\left|\cos \theta_{\mathrm{c} . \mathrm{m} .}^{\omega}\right|<0.8$, there are several prominent features present in the data. Near threshold, the transverse cross section is mostly flat. Around $W \sim 1.9 \mathrm{GeV}$ it begins to develop a humped shape and by $W \sim 2.1 \mathrm{GeV}$ the cross section has two dips. In a concurrent article, we present partial wave analysis results obtained from this data which attribute these 


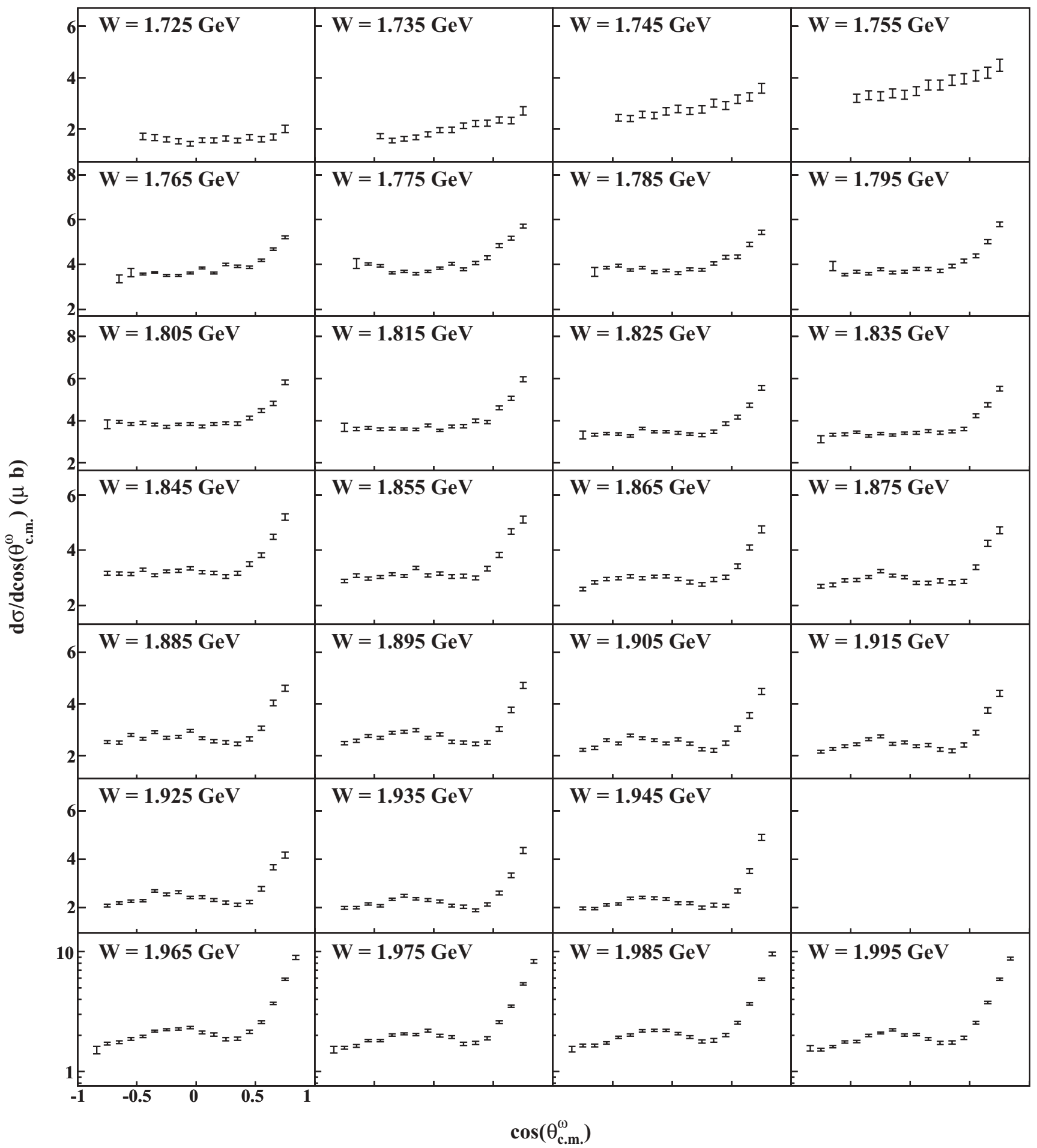

FIG. 7. $\frac{d \sigma}{d \cos \theta_{\text {c.m. }}^{\omega}}(\mu b)$ versus $\cos \theta_{\text {c.m. }}^{\omega}$ : Differential cross-section results for bins in the energy range $1.72 \mathrm{GeV} \leqslant W<2.00 \mathrm{GeV}$. The centroid of each $10-\mathrm{MeV}$ wide bin is printed on the plot. The lack of reported data points in the $W=1.955 \mathrm{GeV}$ bin is discussed in Sec. VI. The error bars, which do not include systematic uncertainties, are discussed in the text. The additional near-threshold background separation uncertainties, discussed in Sec. IV, are clearly visible in the first four center-of-mass energy bins. Note that the vertical scales are linear up to $W$ of $1.945 \mathrm{GeV}$ and logarithmic above that.

features to various baryon resonance contributions [16]. For now, we simply aim to draw attention to some of the prominent structures present in our measurements.
A very prominent forward peak begins to rise just above threshold and continues to be the dominant feature of the cross section up through our highest energies. This type of 


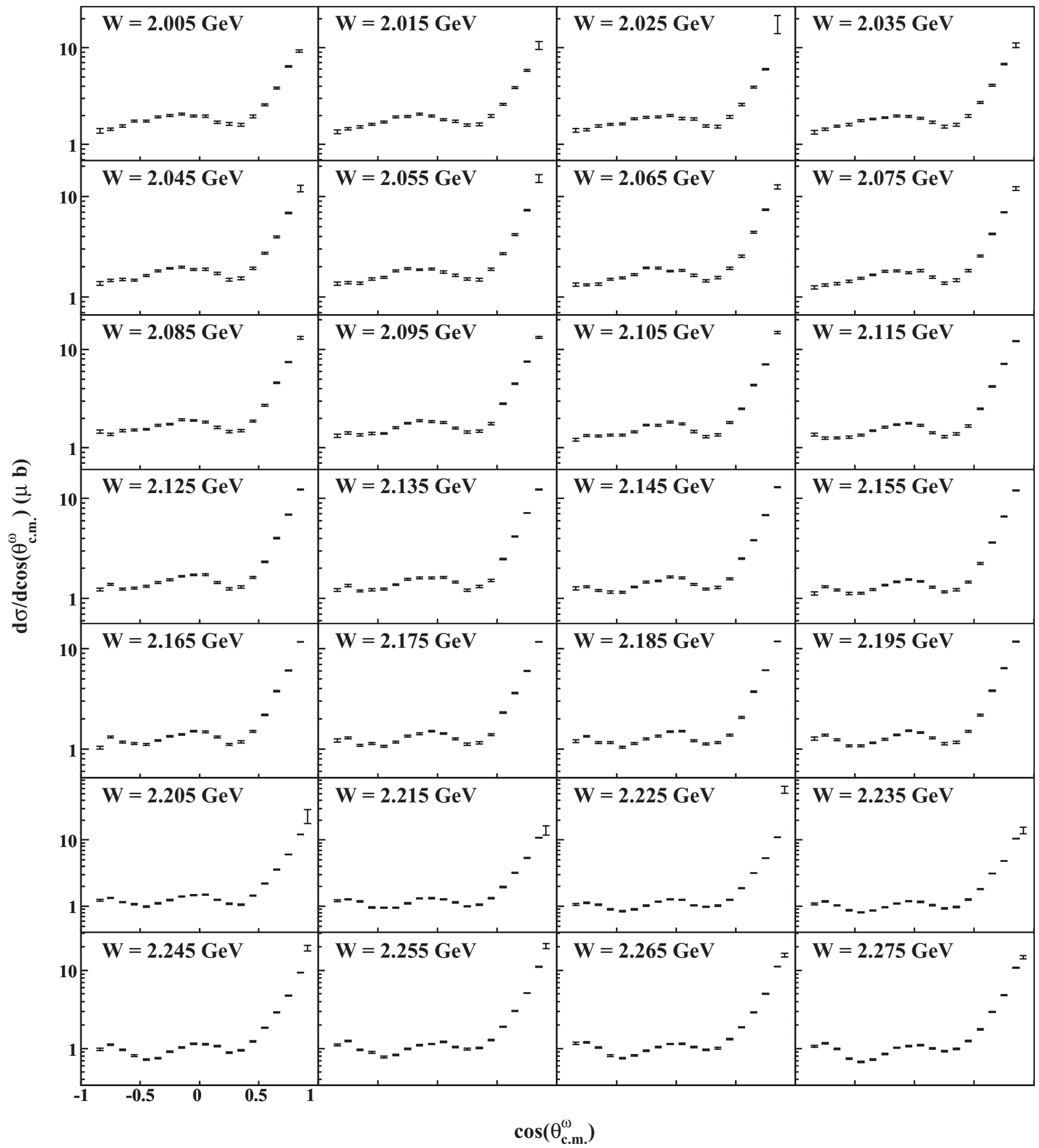

FIG. 8. $\frac{d \sigma}{d \cos \theta_{\mathrm{cm}}^{\omega}}(\mu \mathrm{b})$ versus $\cos \theta_{\mathrm{c} . \mathrm{m}}^{\omega}$ : Differential cross-section results for bins in the energy range $2.00 \mathrm{GeV} \leqslant W<2.28 \mathrm{GeV}$. The centroid of each 10-MeV wide bin is printed on the plot. The error bars, which do not include systematic uncertainties, are discussed in the text.

behavior typically indicates the presence of strong $t$-channel contributions. Models of $\omega$ photoproduction, e.g., Refs. [6-9], typically associate this peak with $t$-channel contributions from $\pi^{0}, \eta$, and Pomeron exchange. A backward peak begins to emerge around $W \sim 2.2 \mathrm{GeV}$, whose prominence increases as the energy increases (although it is always at least one order of magnitude smaller than the forward peak). This could be indicative of the presence of contributions in the $u$ channel. Many models of this reaction attribute this peak to $u$-channel nucleon exchange [6-9]; however, comparisons 


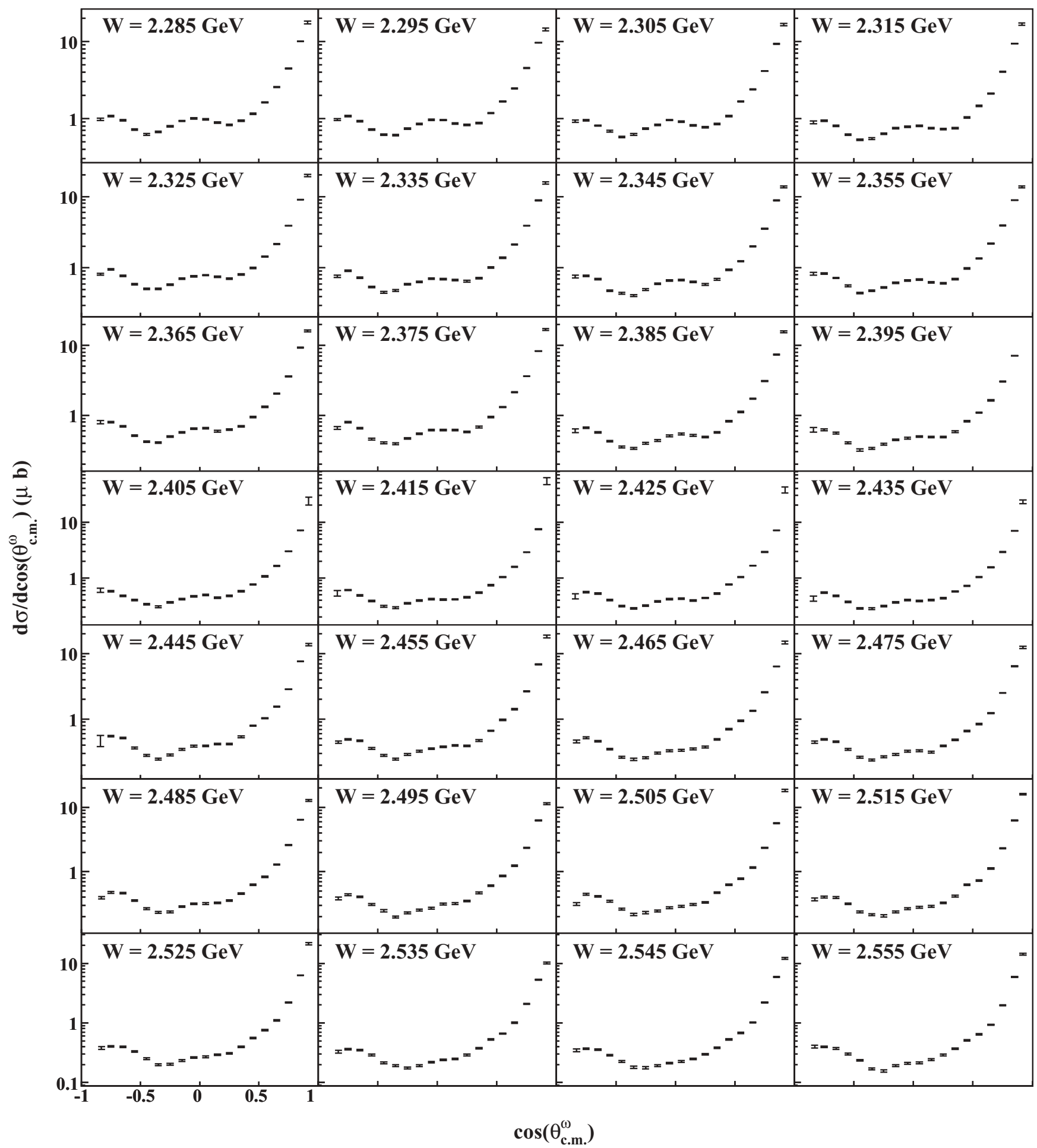

FIG. 9. $\frac{d \sigma}{d \cos \theta_{\mathrm{cm}}^{\omega}}(\mu \mathrm{b})$ versus $\cos \theta_{\mathrm{c} . \mathrm{m}}^{\omega}$ : Differential cross-section results for bins in the energy range $2.28 \mathrm{GeV} \leqslant W<2.56 \mathrm{GeV}$. The centroid of each 10-MeV wide bin is printed on the plot. The error bars, which do not include systematic uncertainties, are discussed in the text.

of the spin density matrix elements predicted by these models to the new high-precision measurements presented in this article casts doubt on the validity of these models (see Sec. VIII C).

\section{B. Spin density matrix elements}

The polarization of the $\omega$ can be studied by examining the distributions of its decay products. Since the $\omega$ is a spin-1 particle, its spin density matrix has nine complex elements; 


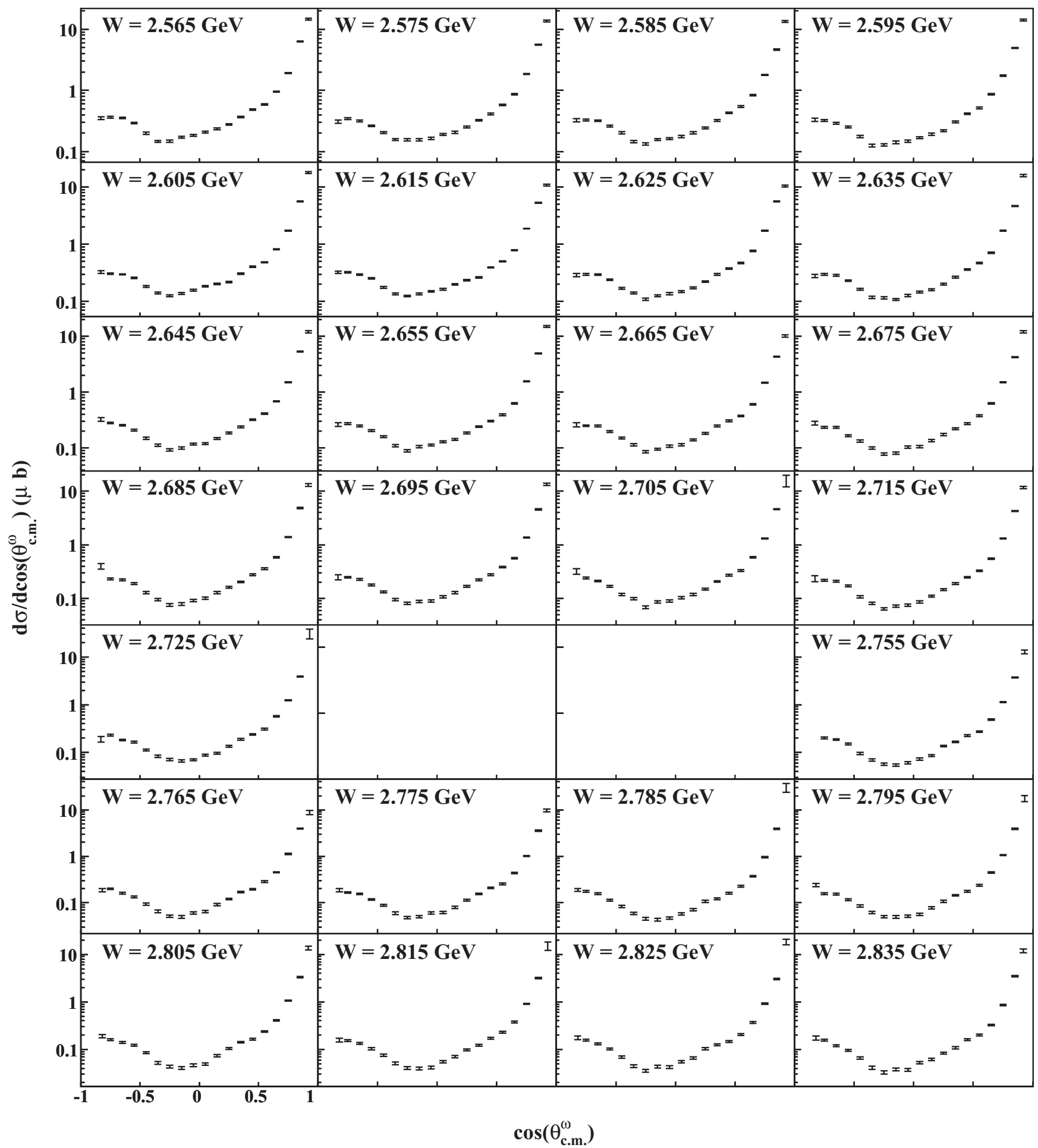

FIG. 10. $\frac{d \sigma}{d \cos \theta_{\mathrm{cm}}^{\omega}}(\mu \mathrm{b})$ versus $\cos \theta_{\mathrm{c} . \mathrm{m}}^{\omega}$ : Differential cross-section results for bins in the energy range $2.56 \mathrm{GeV} \leqslant W<2.84 \mathrm{GeV}$. The centroid of each $10-\mathrm{MeV}$ wide bin is printed on the plot. The lack of reported data points in the $W=2.735 \mathrm{GeV}$ and $W=2.745 \mathrm{GeV}$ bins is discussed in Sec. VI. The error bars, which do not include systematic uncertainties, are discussed in the text.

however, parity, hermiticity, and normalization reduce the number of independent elements (for an unpolarized beam) to four real quantities (of which, three are measurable). Traditionally, these are chosen to be $\rho_{00}^{0}, \rho_{1-1}^{0}$, and $\operatorname{Re}\left(\rho_{10}^{0}\right)$. Our results cover a large range of energies and angles; thus, we chose the quantization axis to be the photon direction in the overall c.m. frame, known as the Adair frame [24].

The spin-density matrix elements can be written in terms of the production amplitudes $\mathcal{A}$ (i.e., the scattering 


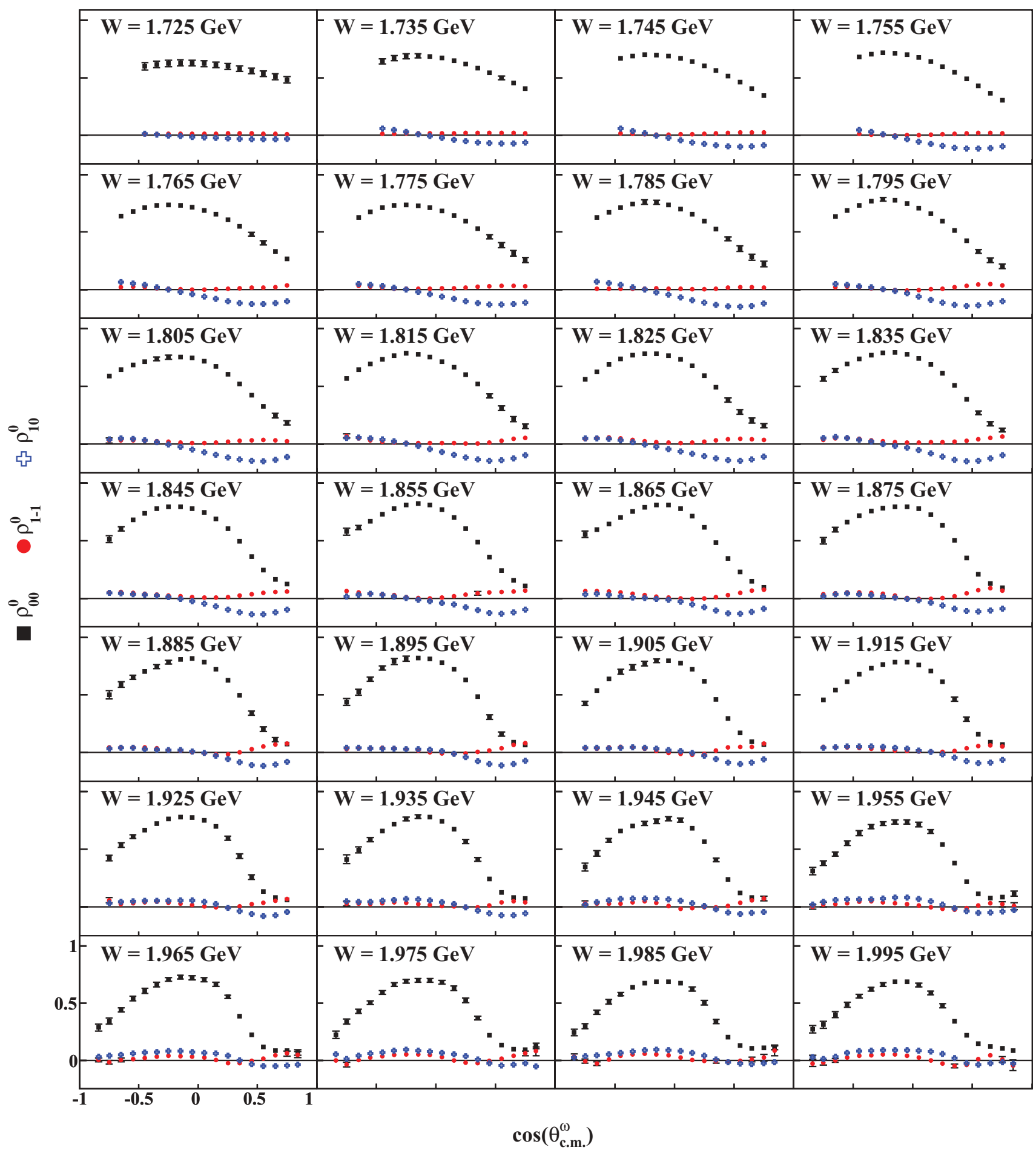

FIG. 11. (Color online) $\rho_{M M^{\prime}}^{0}$ versus $\cos \theta_{\text {c.m. }}^{\omega}$ : Spin-density matrix element measurements, in the Adair frame, for bins in the range $1.72 \mathrm{GeV} \leqslant W<2.00 \mathrm{GeV}$. The black squares are $\rho_{00}^{0}$, the (red) circles are $\rho_{1-1}^{0}$, and the (blue) crosses are $\operatorname{Re}\left(\rho_{10}^{0}\right)$. The centroid of each $10-\mathrm{MeV}$ wide bin is printed on the plot. The error bars do not include systematic uncertainties.

amplitudes $\mathcal{M}$ introduced in Sec. $V$ without the $\omega$ decay piece), as

$$
\rho_{M M^{\prime}}^{0}=\frac{1}{N} \sum_{m_{\gamma}, m_{i}, m_{f}} \mathcal{A}_{m_{i}, m_{\gamma}, m_{f}, M} \mathcal{A}_{m_{i}, m_{\gamma}, m_{f}, M^{\prime}}^{*}
$$

where the $M, M^{\prime}$ refer to the spin projection of the $\omega$ (on the photon direction in the c.m. frame) and

$$
N=\sum_{m_{i}, m_{\gamma}, m_{f}} \sum_{M}\left|\mathcal{A}_{m_{i}, m_{\gamma}, m_{f}, M}\right|^{2}
$$




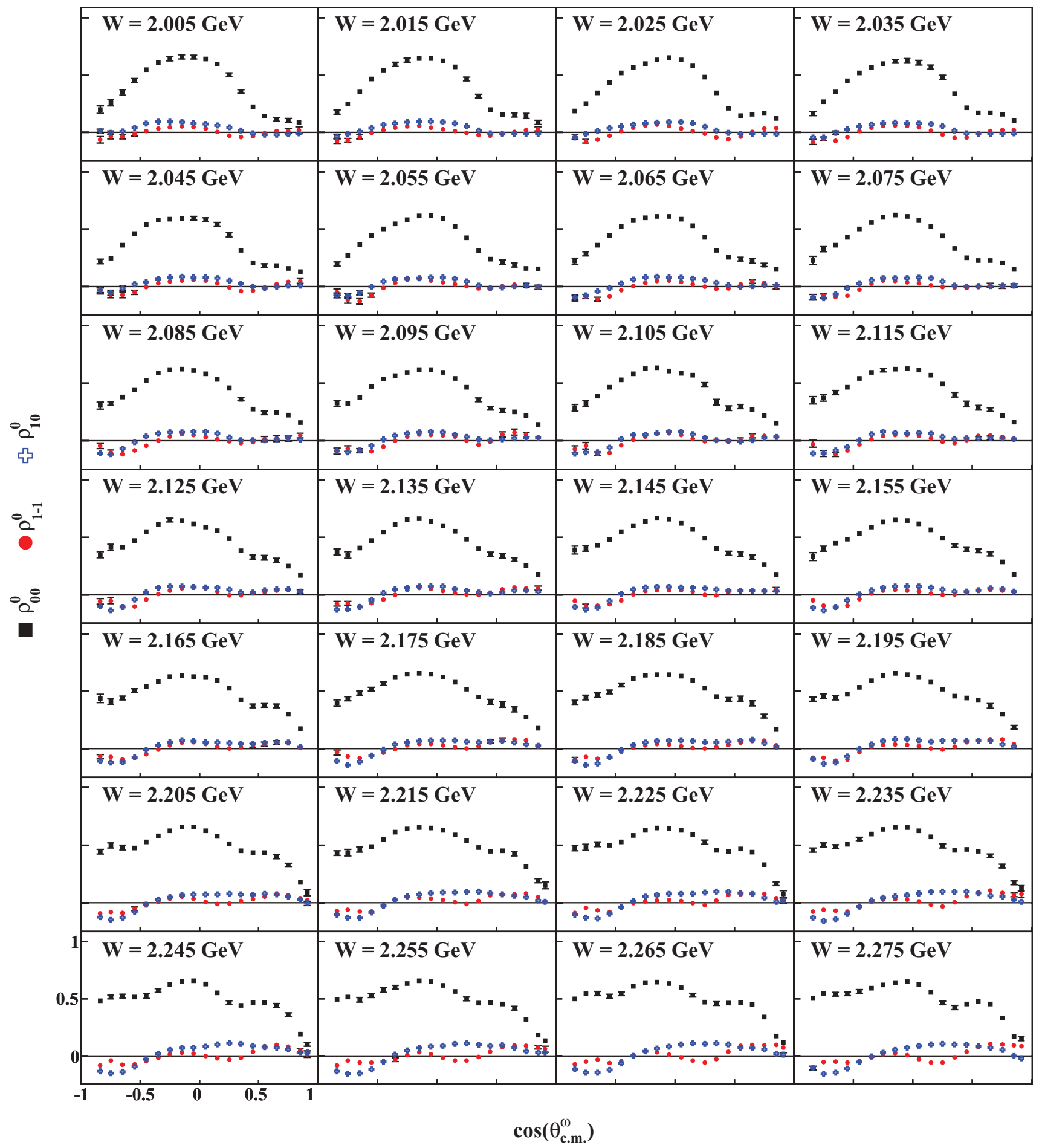

FIG. 12. (Color online) $\rho_{M M^{\prime}}^{0}$ versus $\cos \theta_{\mathrm{c} . \mathrm{m}}^{\omega}$ : Spin-density matrix element measurements, in the Adair frame, for bins in the range $2.00 \mathrm{GeV} \leqslant W<2.28 \mathrm{GeV}$. The black squares are $\rho_{00}^{0}$, the (red) circles are $\rho_{1-1}^{0}$, and the (blue) crosses are $\operatorname{Re}\left(\rho_{10}^{0}\right)$. The centroid of each $10-\mathrm{MeV}$ wide bin is printed on the plot. The error bars do not include systematic uncertainties.

is a normalization factor. Using the production amplitudes obtained from the event-based fits described in Sec. V, the spin density matrix elements were projected out of the partial wave expansion at $2015\left(W, \cos \theta_{\mathrm{c} . \mathrm{m} .}^{\omega}\right)$ points. These data points correspond to the centroids of the bins for which cross-section results are reported, along with additional points in the $W=1.955 \mathrm{GeV}, 2.735 \mathrm{GeV}$, and $2.745 \mathrm{GeV}$ center-of-mass energy bins for which cross-sections results are not reported due to normalization issues (see Sec. VI).

Figures 11-14 show the $\rho_{M M^{\prime}}^{0}$ results extracted using the partial wave expansion technique. The error bars are purely 


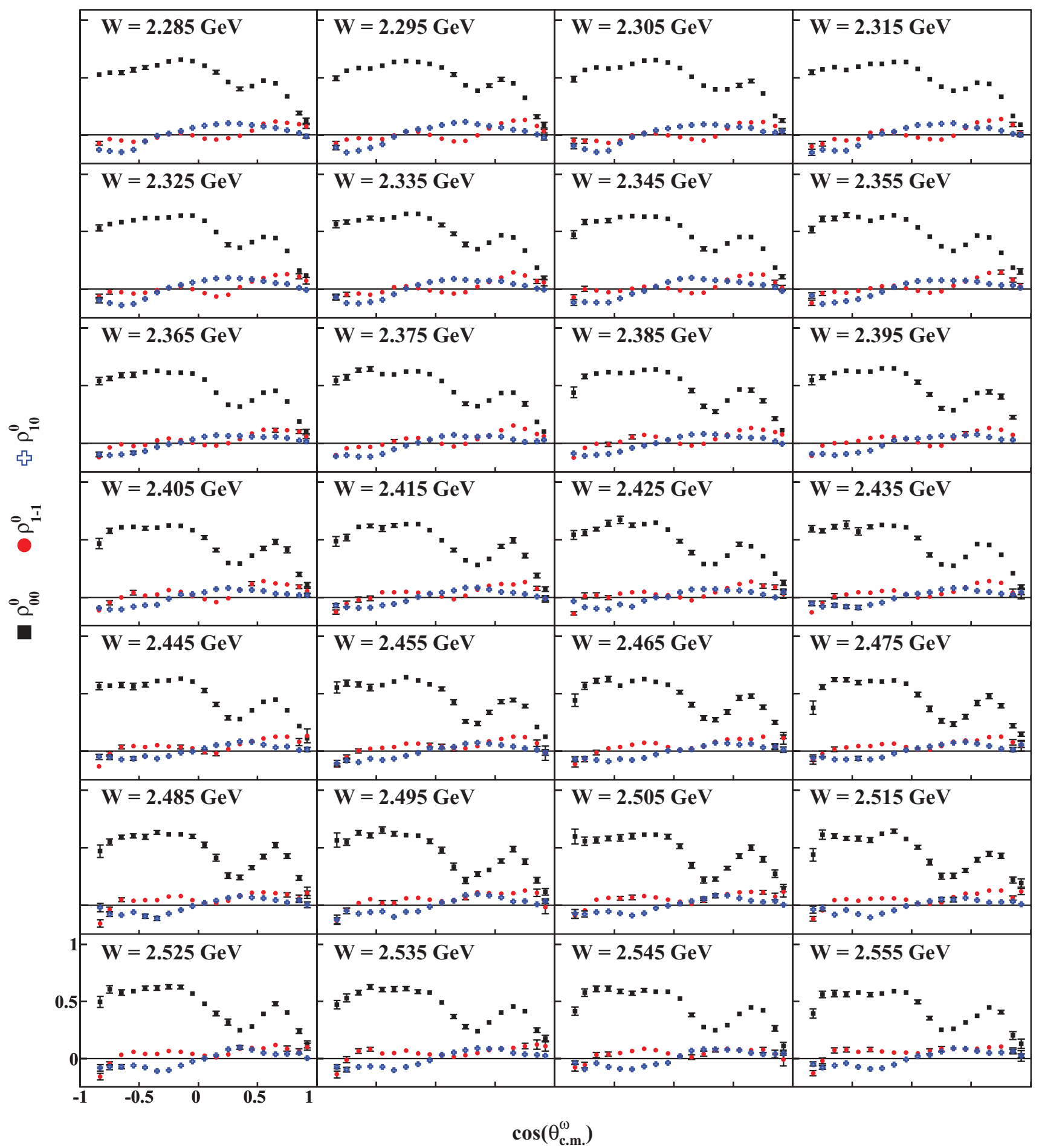

FIG. 13. (Color online) $\rho_{M M^{\prime}}^{0}$ versus $\cos \theta_{\text {c.m. }}^{\omega}$ : Spin-density matrix element measurements, in the Adair frame, for bins in the range $2.28 \mathrm{GeV} \leqslant W<2.56 \mathrm{GeV}$. The black squares are $\rho_{00}^{0}$, the (red) circles are $\rho_{1-1}^{0}$, and the (blue) crosses are $\operatorname{Re}\left(\rho_{10}^{0}\right)$. The centroid of each $10-\mathrm{MeV}$ wide bin is printed on the plot. The error bars do not include systematic uncertainties.

statistical. The spin-density matrix elements do not rely on normalization information; thus, only the acceptance can contribute to the systematic uncertainty. Possible effects due to systematic problems in the acceptance calculation were examined by analyzing decay distributions distorted by our estimated acceptance uncertainty. Based on this study, we estimate the systematic uncertainties in our results to be as follows:

$$
\begin{aligned}
\sigma_{00} & =0.0175, \\
\sigma_{1-1} & =0.0125, \\
\sigma_{10} & =0.01 .
\end{aligned}
$$




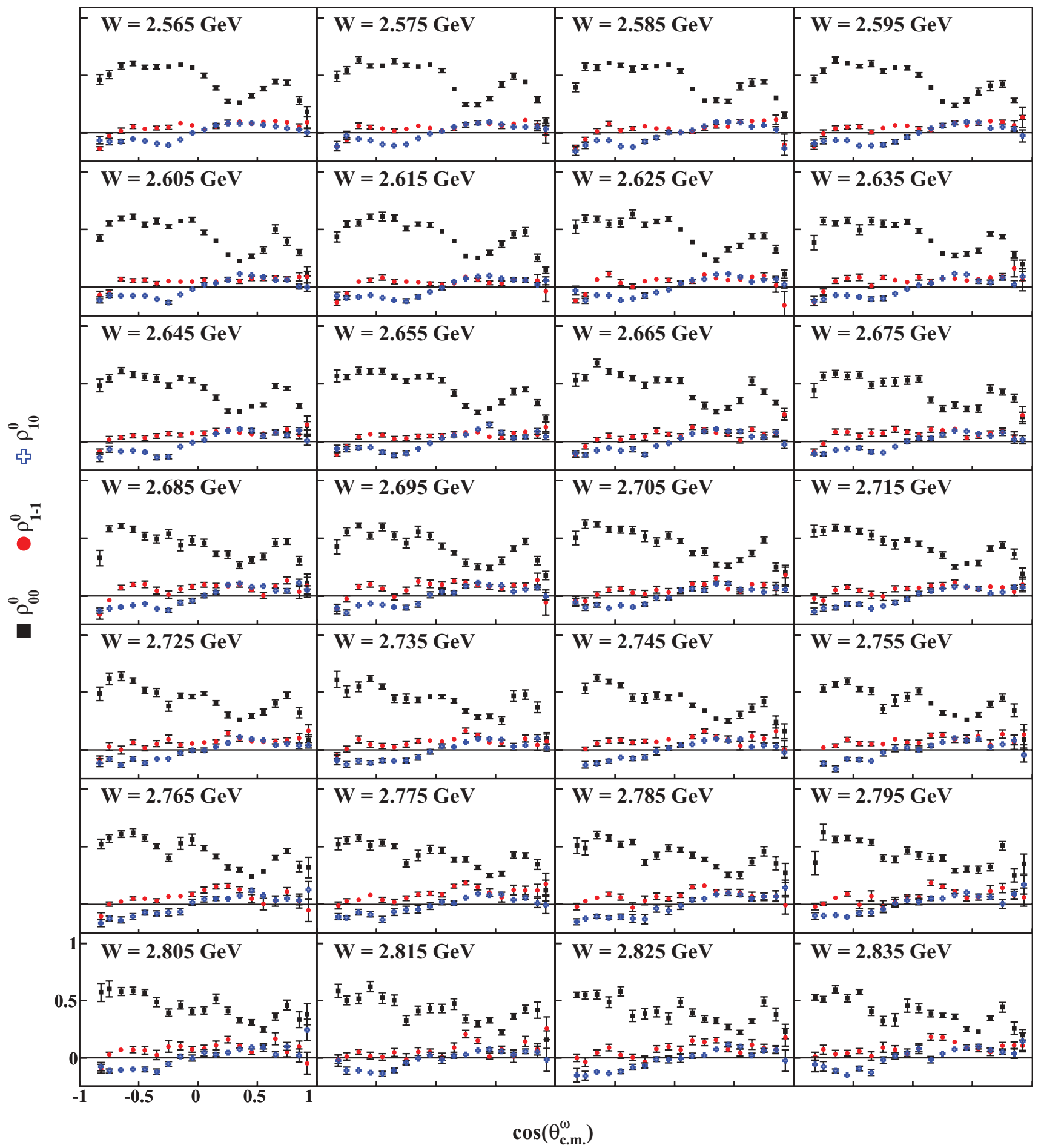

FIG. 14. (Color online) $\rho_{M M^{\prime}}^{0}$ versus $\cos \theta_{\mathrm{c} . \mathrm{m}}^{\omega}$ : Spin-density matrix element measurements, in the Adair frame, for bins in the range $2.56 \mathrm{GeV} \leqslant W<2.84 \mathrm{GeV}$. The black squares are $\rho_{00}^{0}$, the (red) circles are $\rho_{1-1}^{0}$, and the (blue) crosses are $\operatorname{Re}\left(\rho_{10}^{0}\right)$. The centroid of each $10-\mathrm{MeV}$ wide bin is printed on the plot. The error bars do not include systematic uncertainties.

Over most of our kinematics, these results represent the first high-precision measurements of $\rho_{M M^{\prime}}^{0}$ for $\omega$ photoproduction. Near threshold and at forward angles, the cross section develops a strong forward peak, which is indicative of $t$-channel contributions. In this same region, the diagonal $\rho_{00}^{0}$ element decreases sharply as the energy increases, or equivalently, as the forward peak increases in significance. This is typical of exchange of a spin- 0 particle in the $t$-channel where the $\omega$ is forced to carry the spin of the photon at forward angles. This new precise polarization information should help determine the relative strengths of the scalar and pseudoscalar exchanges (see Sec. VIII C). 
At higher energies, starting near $W \sim 2.1 \mathrm{GeV}$, a dip in $\rho_{00}^{0}$ appears at $\cos \theta_{\mathrm{c.m} .}^{\omega} \sim 0.4$, which continues to increase in prominence until about $W \sim 2.5 \mathrm{GeV}$. Above this energy, its significance slowly decreases; however, it is still present at our highest energies. This dip is located near where the forward peak (typically associated with $t$-channel contributions) has decreased in significance such that it is approximately the same size as the cross section in the region $0<\cos \theta_{\text {c.m. }}^{\omega}<0.4$. Thus, it is possible that this dip results from interference between the $t$-channel and larger-angle production mechanisms. In the kinematic regions where the cross section possesses the humps and dips discussed in Sec. VIII A, there are a number of interesting features found in the spin density matrix elements as well. The partial wave analysis we performed on this data found that these features are well described by baryon resonance contributions [16].

\section{Interpretation of the data}

In the low-energy regime, these new measurements have been used to carry out a mass-independent partial wave analysis of the reaction $\gamma p \rightarrow \omega p$. The results of this analysis, which are presented in a concurrent article [16] and are not discussed in detail here, show clear evidence of $s$-channel resonance contributions. This PWA, the results of which are different from previous analyses [8,10-14], was the first to benefit from the strong additional constraints provided by the high-precision polarization results obtained from these data.

The high-energy measurements have been compared to two existing models for $\omega$ photoproduction. The first is the model of Oh et al. [8] which includes pseudoscalar meson $\left(\pi^{0}\right.$ and $\eta$ ) and Pomeron exchange in the $t$ channel, along with nucleon exchange in both the $s$ and $u$ channels. It also includes $s$-channel contributions, which are necessary to describe the data in the central region of the angular range. The second model is that of Laget [6,7] which includes $t$ - and $u$-channel contributions similar to that of Ref. [8], but also allows for a contribution from two-gluon exchange. In this latter model, the two-gluon term is required to describe the $\phi$ photoproduction data.

Figure 15 shows comparisons of these models to our data at $W=2.8 \mathrm{GeV}$. Both models do a reasonable job of reproducing the cross-section measurements. The $t$-channel terms drive the very forward-angle data where the agreement is very good. At backward angles, where the $u$-channel terms dominate, the agreement is not as good as it is at forward angles. In the central region, both models agree with the overall shape of the cross section; however, the finer structure in the data is not reproduced.

Neither model is able to reproduce the new high-precision spin density matrix element measurements presented in this article. While some regions are reasonably well described by one model or the other, neither gives anything close to good overall agreement. Perhaps the most striking discrepancy is that at forward angles, where the cross sections are described very well by both models, neither provides an excellent description of the spin density matrix elements. The

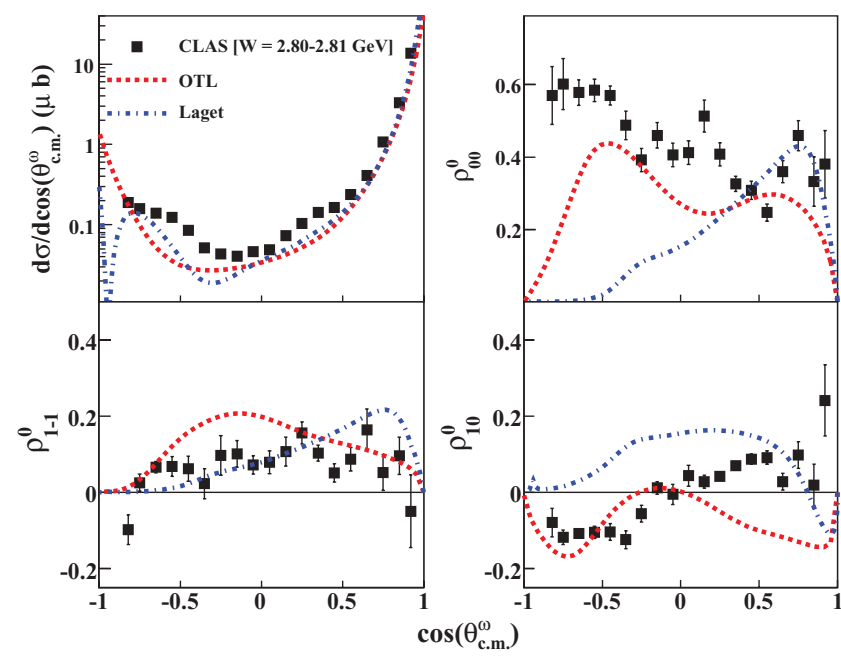

FIG. 15. (Color online) A comparison of the theoretical models of Oh et al. [8] (red, dashed lines) and Laget [6,7] (blue, dot-dashed lines) to the $W=2.805 \mathrm{GeV}$ data presented in this article (black squares).

high-precision measurements presented in this article clearly provide new stringent constraints on both the nature of the production mechanisms in the high-energy regime, as well as on the search for missing baryon resonances.

\section{Comparison to previous measurements}

Previous experimental measurements that overlap our energy range have been made at CLAS in 2003 [4], at SAPHIR

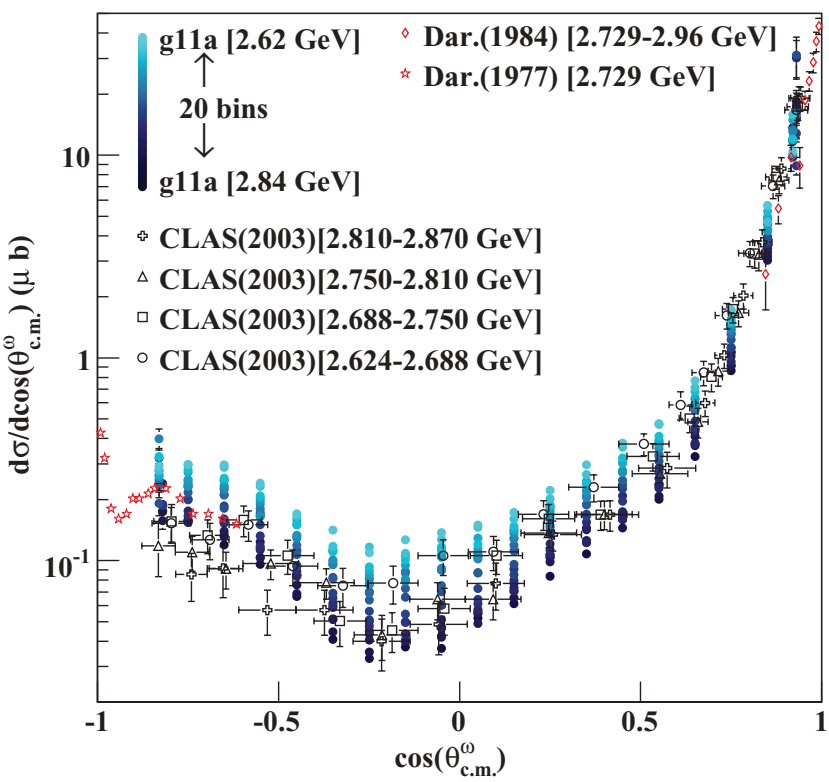

FIG. 16. (Color online) $\frac{d \sigma}{d \cos \theta_{\mathrm{cm}}^{\omega}}(\mu \mathrm{b})$ versus $\cos \theta_{\mathrm{c.m}}^{\omega}$ : Comparison of the present CLAS results [(blue) circles] with previously published results from CLAS [4] (black open crosses, triangles, squares, and circles) and Daresbury [2,3] [(red) open diamonds and stars] in the energy range $2.62 \mathrm{GeV}<W<2.96 \mathrm{GeV}$. The Daresbury (1977) points have no error bars; the points were extracted from a portable document format (PDF) image. 


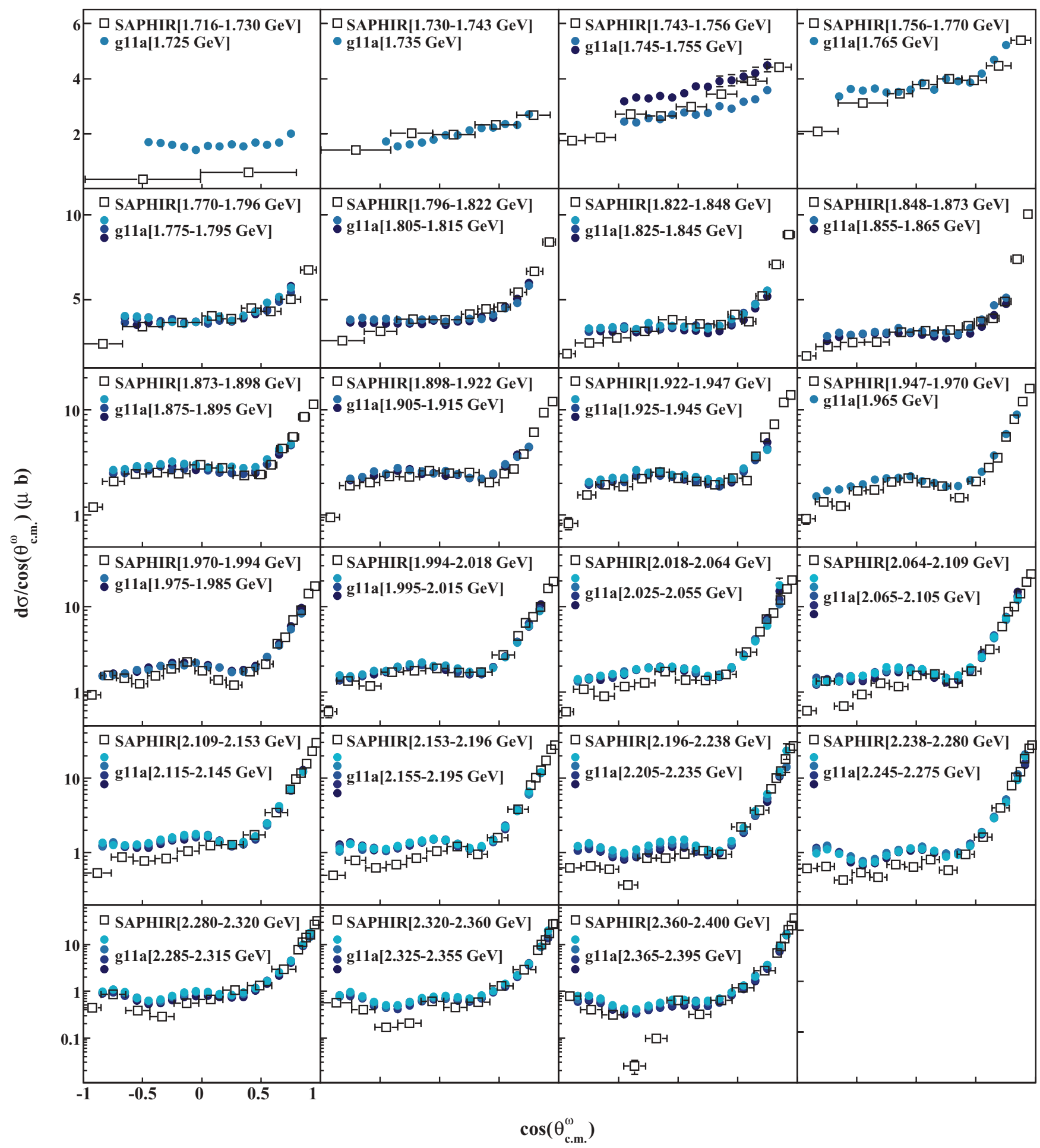

FIG. 17. (Color online) $\frac{d \sigma}{d \cos \theta_{\mathrm{c} . \mathrm{m} .}^{\omega}}(\mu \mathrm{b})$ versus $\cos \theta_{\mathrm{c} . \mathrm{m}}^{\omega}$ : Comparison of the present CLAS results [(blue) circles] with previously published results from SAPHIR [5] (black open squares).

in 2003 [5], at Daresbury in 1984 [3] and 1977 [2], and at SLAC in 1973 [1]. Below we compare our measurements with each of these previous results. The cross sections will be examined first, followed by the spin density matrix elements.

Figure 16 shows a comparison of the cross-section results presented in this article with previously published results from
CLAS [4] and Daresbury [2,3]. The previous CLAS results, four energy bins in the range $2.624 \mathrm{GeV}<W<2.87 \mathrm{GeV}$, cover virtually the same angular range as the current results. The agreement is very good for $\cos \theta_{\mathrm{c} . \mathrm{m} .}^{\omega}>-0.1$; however, there is a sizable discrepancy in the backward direction. At the time of the earlier CLAS measurement, the $\omega$ polarization 

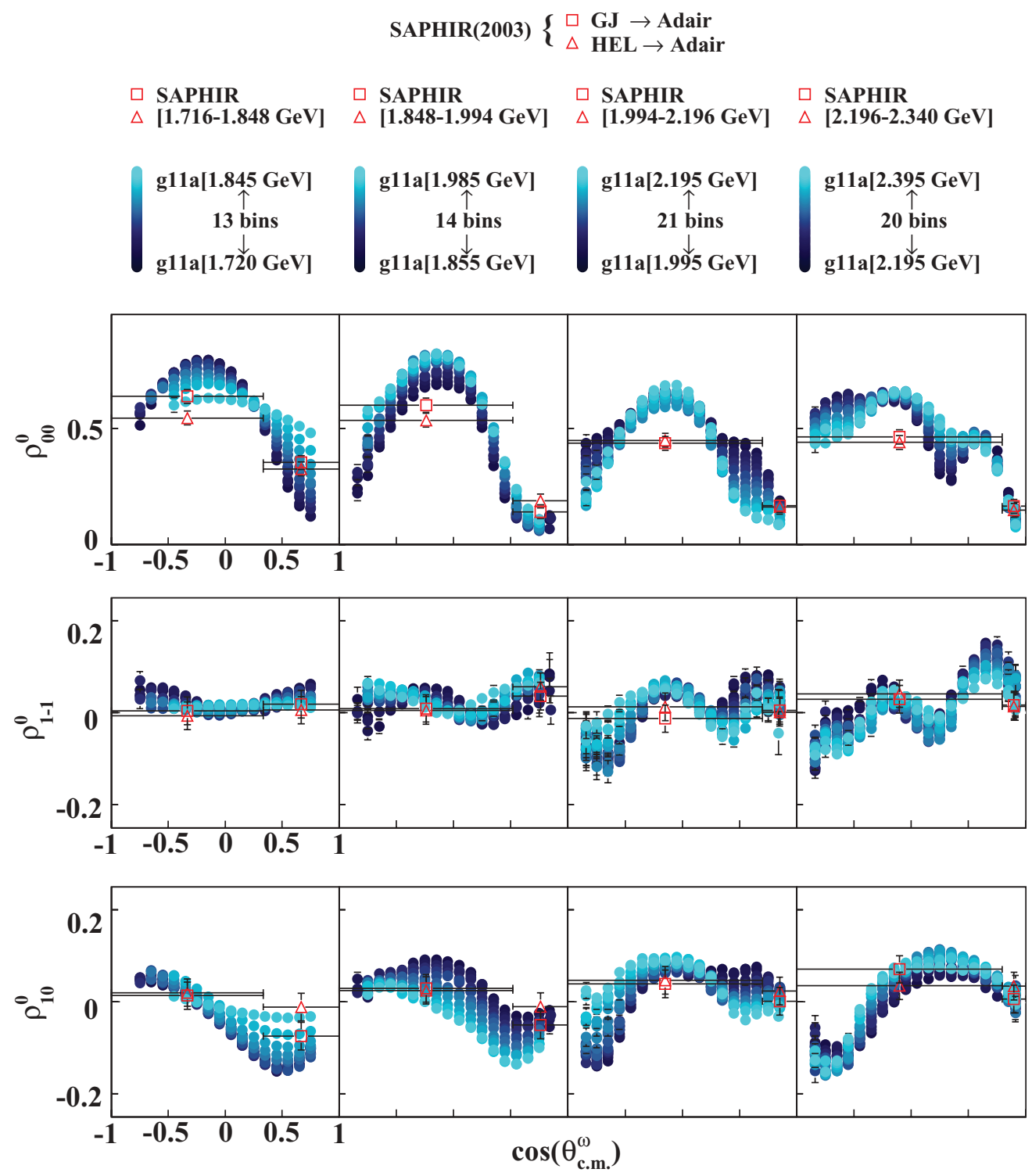

FIG. 18. (Color online) $\rho_{M M^{\prime}}^{0}$ in the Adair frame versus $\cos \theta_{\mathrm{c.m}}^{\omega}$ : Comparison of the present CLAS results [(blue) circles] with previously published SAPHIR [5] results [open (red) squares and triangles]. SAPHIR extracted results independently in the Gottfried-Jackson and helicity frames-both presented here rotated to the Adair frame.

had only been measured in the forward direction (see Fig. 19); thus, these values of the spin density matrix elements were used in the acceptance calculation. Our results show that the polarization is quite different at backward and forward angles. Near the edges of the CLAS acceptance, e.g., in the backward direction, an incorrect description of the polarization can lead to large errors in the acceptance calculation. This is most likely the cause of the discrepancy in the cross sections. The Daresbury results, which were only published in the very forward and backward regions, are in good agreement with our measurements.

For $W<2.4 \mathrm{GeV}$, the previous large acceptance results come from SAPHIR [5]. Figure 17 shows a comparison of the SAPHIR cross-section measurements with the present CLAS results. The error bars shown for the SAPHIR points do not include systematic uncertainties. The agreement is fair, but there are some discrepancies. The SAPHIR experiment had better angular coverage; however, the CLAS results are more precise. In the forward direction, the agreement is very good at all energies. At moderate angles, $\left|\cos \theta_{\text {c.m. }}^{\omega}\right|<0.5$, the agreement is good at lower energies but the CLAS results tend to be higher as the energy increases. In the backward direction, where the CLAS has acceptance, the CLAS points are almost always higher than the SAPHIR points.

Previous spin-density matrix element measurements are much less precise. The only results published for $W<2.4 \mathrm{GeV}$ come from SAPHIR [5]. Figure 18 shows a comparison of the SAPHIR results, which consist only of four energy bins, each with two angular points, and the present CLAS results, which include 1181 total data points in this 
○ Daresbury (1984)[2.477-2.729 GeV]

$\triangle$ Daresbury (1984)[2.729-2.960 GeV] $\mathrm{g} 11 \mathrm{a}(2.475 \mathrm{GeV})$
$\uparrow$
37 bins
$\downarrow$
$\mathrm{g} 11 \mathrm{a}(2.835 \mathrm{GeV})$

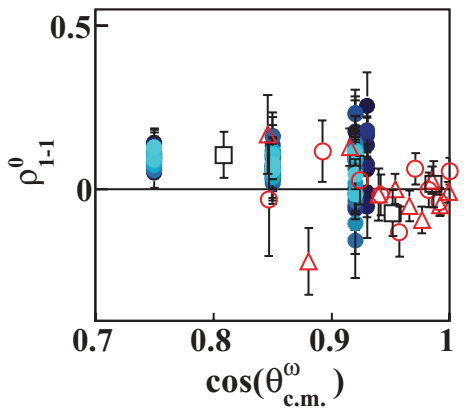

SLAC (1973)[2.477 GeV]

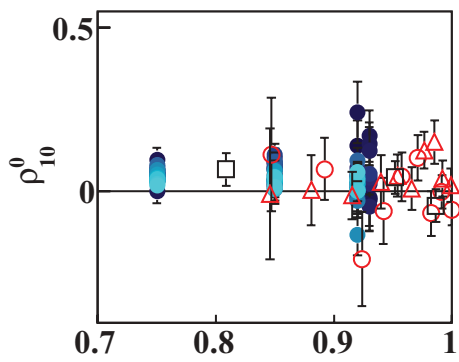

FIG. 19. (Color online) $\rho_{M M^{\prime}}^{0}$ in the Adair frame versus $\cos \theta_{\text {c.m. }}^{\omega}$ : Comparison of the present CLAS results [(blue) circles] with previously published Daresbury [3] [open (red) circles and triangles] and SLAC [1] (open black squares).

energy range. We note here that the SAPHIR Collaboration published their results in both the Gottfried-Jackson and helicity frames, with each measurement constituting an independent fit to their data. Both results were rotated into the Adair frame for comparison. Overall, the SAPHIR results are in good agreement with our measurements.

At higher energies, previously published results only exist at very forward angles. Figure 19 shows a comparison of our forward high energy results with those from Daresbury [3] and SLAC [1]. The agreement is good. For $W>2.4 \mathrm{GeV}$, the results presented in this article for $\rho_{M M^{\prime}}^{0}$ are the world's first measurements for $\cos \theta_{\mathrm{c} . \mathrm{m} .}^{\omega}<0.8$.

\section{CONCLUSIONS}

In summary, experimental results for $\omega$ photoproduction from the proton have been presented in the energy regime from threshold up to $W=2.84 \mathrm{GeV}$. Both differential cross section and spin-density matrix element measurements are reported. The cross-section results are the most precise to date and provide the largest energy and angular coverage. The results are in fair to good agreement with previous experiments. For $W<2.4 \mathrm{GeV}$, we present $1181 \rho_{M M^{\prime}}^{0}$ data points; the previous world's data consisted of eight points. At higher energies, we have made the first spin density matrix element measurements for $\cos \theta_{\mathrm{c} . \mathrm{m} .}^{\omega}<0.8$. Our $\rho_{M M^{\prime}}^{0}$ measurements are in good agreement with the, rather sparse, existing data. The $1960\left(W, \cos \theta_{\text {c.m. }}^{\omega}\right)$ cross-section points, along with the 2015 $\left(W, \cos \theta_{\text {c.m. }}^{\omega}\right)$ spin-density matrix element data points can be obtained at Ref. [25].
These new data will have a large impact on our current understanding of vector-meson photoproduction, as well as provide a crucial data set in the search for missing baryon resonances. A mass-independent partial wave analysis performed on these data, which is the first such analysis to benefit from the strong constraints provided by high-precision polarization information, found strong evidence for baryon resonance contributions [16]. Furthermore, none of the current models of high-energy $\omega$ photoproduction are able to describe the precise spin density matrix element measurements presented in this article. We look forward to seeing what impact these new results will have on future models of vector-meson photoproduction.

\section{ACKNOWLEDGMENTS}

We thank the staff of the Accelerator and the Physics Divisions at Thomas Jefferson National Accelerator Facility who made this experiment possible. This work was supported in part by the US Department of Energy (under Grant No. DE-FG02-87ER40315), the National Science Foundation, the Italian Istituto Nazionale di Fisica Nucleare, the French Centre National de la Recherche Scientifique, the French Commissariat à l'Energie Atomique, the Science and Technology Facilities Council (STFC), and the Korean Science and Engineering Foundation. The Southeastern Universities Research Association (SURA) operated Jefferson Lab under US DOE Contract No. DE-AC05-84ER40150 during this work.
[1] J. Ballam et al., Phys. Rev. D 7, 3150 (1973).

[2] R. W. Clift et al., Phys. Lett. B72, 144 (1977).

[3] D. P. Barber et al. (LAMP2 Group Collaboration), Z. Phys. C 26, 343 (1984).

[4] M. Battaglieri et al. (CLAS Collaboration), Phys. Rev. Lett. 90, 022002 (2003).

[5] J. Barth et al. (SAPHIR Collaboration), Eur. Phys. J. A 18, 117 (2003).
[6] J.-M. Laget, Phys. Lett. B489, 313 (2000).

[7] J.-M. Laget, Nucl. Phys. A699, 184c (2002).

[8] Y. Oh, A. I. Titov, and T.-S. H. Lee, Phys. Rev. C 63, 025201 (2001).

[9] A. Sibirtsev, K. Tsushima, and S. Krewald, arXiv:nuclth/0202083 (2002).

[10] M. W. Paris, Phys. Rev. C 79, 025208 (2009).

[11] Q. Zhao, Phys. Rev. C 63, 025203 (2001). 
[12] A. I. Titov and T.-S. H. Lee, Phys. Rev. C 66, 015204 (2002).

[13] G. Penner and U. Mosel, Phys. Rev. C 66, 055212 (2002).

[14] V. Shklyar, H. Lenske, U. Mosel, and G. Penner, Phys. Rev. C 71, 055206 (2005).

[15] S. Capstick and W. Roberts, Phys. Rev. D 49, 4570 (1994).

[16] M. Williams et al. (CLAS Collaboration), Phys. Rev. C 80, 065209 (2009).

[17] M. Williams et al., "The impact on the phenomenology of $\gamma p \rightarrow$ $p \omega$ of the first high-precision measurements of $\omega$ polarization observables," Phys. Rev. (to be submitted).

[18] D. I. Sober et al., Nucl. Instrum. Methods Phys. Res. A 440, 263 (2000).
[19] B. A. Mecking et al., Nucl. Instrum. Methods Phys. Res. A 503, 513 (2003).

[20] G. J. Feldman and R. D. Cousins, Phys. Rev. D 57, 3873 (1998).

[21] M. Williams, Ph.D. thesis, Carnegie Mellon University, 2007, www.jlab.org/Hall-B/general/clas_thesis.html.

[22] M. Williams, M. Bellis, and C. A. Meyer, JINST 4, P10003 (2009).

[23] J. Ball and E. Pasyuk, CLAS Note 2005-002 (2005), www.jlab.org/Hall-B/notes/.

[24] K. Schilling, P. Seyboth, and G. Wolf, Nucl. Phys. B15, 397 (1970).

[25] The CLAS physics database, clasweb.jlab.org/physicsdb. 